This is the accepted manuscript made available via CHORUS. The article has been published as:

Conservation laws and evolution schemes in geodesic, hydrodynamic, and magnetohydrodynamic flows

Charalampos Markakis, Kōji Uryū, Eric Gourgoulhon, Jean-Philippe Nicolas, Nils

Andersson, Athina Pouri, and Vojtěch Witzany

Phys. Rev. D 96, 064019 - Published 13 September 2017

DOI: 10.1103/PhysRevD.96.064019 


\title{
Conservation laws and evolution schemes in geodesic, hydrodynamic and magnetohydrodynamic flows
}

\author{
Charalampos Markakis, 1,2 , 田 Kōji Uryū, 3, 团 Eric Gourgoulhon, 4, 团 Jean-Philippe \\ Nicolas, ${ }^{5,8}$ Nils Andersson, ${ }^{2}$, I Athina Pouri, ${ }^{6, * * *}$ and Vojtěch Witzany ${ }^{7},{ }^{+}$ \\ ${ }^{1}$ NCSA, University of Illinois at Urbana-Champaign, IL 61801, USA \\ ${ }^{2}$ Mathematical Sciences, University of Southampton, Southampton SO17 1BJ, United Kingdom \\ ${ }^{3}$ Department of Physics, University of the Ryukyus, Senbaru, Nishihara, Okinawa 903-0213, Japan \\ ${ }^{4}$ LUTh, UMR 8102 du CNRS, Observatoire de Paris, \\ Université Paris Diderot, F-92190 Meudon, France \\ ${ }^{5}$ Département de Mathématiques, Université de Bretagne Occidentale \\ 6 avenue Victor Le Gorgeu, 29238 Brest Cedex 3, France \\ ${ }^{6}$ RCAAM, Academy of Athens, Soranou Efesiou 4, 11527, Athens, Greece \\ ${ }^{7}$ ZARM, Universität Bremen, Am Fallturm, 28359 Bremen, Germany
}

(Dated: 29 December 2016)

\begin{abstract}
Carter and Lichnerowicz have established that barotropic fluid flows are conformally geodesic and obey Hamilton's principle. This variational approach can accommodate neutral, or charged and poorly conducting, fluids. We show that, unlike what has been previously thought, this approach can also accommodate perfectly conducting magnetofluids, via the Bekenstein-Oron description of ideal magnetohydrodynamics. When Noether symmetries associated with Killing vectors or tensors are present in geodesic flows, they lead to constants of motion polynomial in the momenta. We generalize these concepts to hydrodynamic flows. Moreover, the Hamiltonian descriptions of ideal magnetohydrodynamics allow one to cast the evolution equations into a hyperbolic form useful for evolving rotating or binary compact objects with magnetic fields in numerical general relativity. In this framework, Ertel's potential vorticity theorem for baroclinic fluids arises as a special case of a conservation law valid for any Hamiltonian system. Moreover, conserved circulation laws, such as those of Kelvin, Alfvén and Bekenstein-Oron, emerge simply as special cases of the Poincaré-Cartan integral invariant of Hamiltonian systems. We use this approach to obtain an extension of Kelvin's theorem to baroclinic (non-isentropic) fluids, based on a temperature-dependent time parameter. We further extend this result to perfectly or poorly conducting baroclinic magnetoflows. Finally, in the barotropic case, such magnetoflows are shown to also be geodesic, albeit in a Finsler (rather than Riemann) space.
\end{abstract}

PACS numbers: 04.40.Nr, 47.10.ab, 47.10.Df, 47.10.A-

\section{INTRODUCTION}

A wide variety of compact stellar objects where general relativistic effects are important is currently known. Black holes and neutron stars are involved in many astrophysical phenomena, including binary mergers and gamma ray bursts, which have observable imprints in the electromagnetic and gravitational wave spectrum. Many of these phenomena can be explained by means of general relativistic hydrodynamics. In addition, there is a growing number of observed phenomena where electromagnetic effects play a major role. These include observations of accretion disks around black holes [1], jets in active galactic nuclei or microquasars [2, 3], gamma ray bursts, hypernovae, pulsars [4] and magnetars [5-

\footnotetext{
* markakis@illinois.edu

$\dagger$ uryu@sci.u-ryukyu.ac.jp

‡ eric.gourgoulhon@obspm.fr

$\S$ jean-philippe.nicolas@univ-brest.fr

ฯ N.A.Andersson@soton.ac.uk

** athpouri@phys.uoa.gr

†† witzany@zarm.uni-bremen.de
}

9]. Magnetohydrodynamics (MHD) provides a macroscopic continuum approximation to studying such phenomena. General relativistic magnetohydrodynamics (GRMHD) originates in the works of Lichnerowicz 10] and is a rapidly developing field of modern astrophysics 4, 11, 12]. Departures from MHD are discussed in [1315] and references therein. Compact objects such as magnetars or the differentially rotating supramassive remnants of binary neutron-star mergers can have magnetic fields of the order of $10^{15}-10^{17} \mathrm{G}$ which can affect the dynamics and stability [16] of these objects. A fully relativistic description of magnetized neutron stars is thus desirable.

In this article, we develop a geometric treatment of ideal GRMHD. To this aim, we use Cartan's exterior calculus, relying on the nature of the electromagnetic field as a 2 -form and the well known formulation of Maxwell's equations by means of the exterior derivative operator. We also employ the formulation of hydrodynamics in terms of the fluid vorticity 2 -form, following Synge [17] and Lichnerowicz [18]. This enables us to formulate GRMHD entirely in terms of exterior forms. Such an approach is not only elegant and fully covariant, but also simplifies some calculations which are tedious in the 
component approach. In addition, we obtain particle-like Lagrangian and Hamiltonian descriptions of ideal MHD, in Newtonian and relativistic contexts, with several theoretical and practical advantages. For example, schemes for evolution in numerical relativity are straightforward to obtain, and conserved quantities whose origin seems ad hoc in the component approach, emerge immediately as Noether-related quantities in this canonical approach.

In particular, Synge and Lichnerowicz have shown that barotropic fluid flows may be described via simple variational principles as geodesic flows in a manifold conformally related to the spacetime manifold. Arnol'd described the nonrelativistic Euler equation as the geodesic equation on the group of volume-preserving diffeomorphisms [19]. This allowed him to apply geometric and group-theoretical methods to the study of this equation, and to develop the now called Arnol'd stability method [20 26].

Carter 27] has used this powerful canonical approach to efficiently derive conservation laws for neutral or charged poorly conducting fluids in general relativity. Markakis [28] has obtained an Euler-Lagrange and a Hamiltonian description of a barotropic fluid valid in Newtonian gravity as well as $3+1$ general relativity. In this article, we extend Carter's framework to perfectly conducting fluids with the aid of the Bekenstein-Oron (hereafter BO) formulation of ideal MHD [29 31]. In the canonical approach, conserved circulation integrals, such as those of Alfvén, Kelvin and Bekenstein-Oron, emerge simply as special cases of the Poincaré-Cartan integral invariant of Hamiltonian systems. We further show that the BO description can describe an arbitrary ideal MHD flow without loss of generality and allows one to cast the ideal MHD equations into a circulation-preserving hyperbolic form, which may be useful in numerical simulations of oscillating stars or radiating binaries with magnetic fields in numerical relativity. We generalize the SyngeLichnerowicz result to perfectly conducting magnetofluids by showing that ideal MHD flows can be described as geodesic flows in a Finsler space.

Finally, Kelvin's circulation theorem has been thought to hold only for barotropic flows. It has been thought not to hold for baroclinic (non-isentropic) flows, except in a weak form (i.e. if the circulation is initially computed along rings of constant temperature or specific entropy [32]). However, using a temperature-dependent time parameter, we obtain a Hamiltonian action principle describing inviscid baroclinic flows within Carter's framework. Moreover, a Poincaré-Cartan integral invariant exists iff a system is Hamiltonian. We thus infer that, contrary to common belief, a generalization of Kelvin's theorem to baroclinic flows does exist in the strong form (i.e. the circulation can be initially computed along an arbitrary fluid ring). Remarkably, this result can be further extended to perfectly or poorly conducting baroclinic magnetoflows.

Symmetries and conservation laws are very useful because they can provide valuable insight of complicated (magneto)hydrodynamic phenomena; the relevant conserved quantities can be extremely useful in constructing initial data in numerical relativity, or significantly simplify solving for the motion. The examples considered below are applicable, among others, to the mathematical study and numerical simulation of fluid motions in rotating or binary relativistic stars 33 -42] and their magnetospheres [43, 44], neutron-star or black-hole accretion rings [27, 45 47] and cosmological dynamics [48 56].

\section{CLASSICAL DYNAMICS IN COVARIANT LANGUAGE}

\section{A. Notation}

We consider a spacetime $(\mathscr{M}, \boldsymbol{g})$, i.e. a fourdimensional real manifold $\mathscr{M}$ endowed with a Lorentzian metric $\boldsymbol{g}$ of signature $(-+++)$. We assume that $\mathscr{M}$ is orientable, so that we have at our disposal the LeviCivita tensor $\boldsymbol{\epsilon}$ (also called volume element) associated with the metric $\boldsymbol{g}$. Let $\boldsymbol{\nabla}$ be the covariant derivative associated with $\boldsymbol{g}: \boldsymbol{\nabla} \boldsymbol{g}=0$ and $\boldsymbol{\nabla} \boldsymbol{\epsilon}=0$. The star operator $\star$ denotes the Hodge dual of a differential form. For example, the Hodge dual of the 1 -form $\boldsymbol{\omega}$ is a 3 -form denoted by $\star \omega$ :

$$
\star \omega_{\alpha \beta \gamma}:=\epsilon_{\alpha \beta \gamma \delta} \omega^{\delta}
$$

Similarly, the Hodge-dual of the 2 -form $\Omega$ is a 2 -form denoted by $\star \Omega$ :

$$
\star \Omega_{\alpha \beta}:=\frac{1}{2} \epsilon_{\alpha \beta \gamma \delta} \Omega^{\gamma \delta}
$$

More details on these definitions may be found e.g. in Appendix B of Ref. 57.

We shall often use an index-free notation, denoting vectors and tensors on $\mathscr{M}$ by boldface symbols. As in [27], given a linear form $\boldsymbol{\omega}$, we denote by $\overrightarrow{\boldsymbol{\omega}}$ the vector associated to it by the metric tensor:

$$
\omega=: \boldsymbol{g}(\overrightarrow{\boldsymbol{\omega}},-\mathbf{-})
$$

In a given vector basis $\left(\boldsymbol{e}_{\alpha}\right)$, the components of $\boldsymbol{g}, \overrightarrow{\boldsymbol{\omega}}$ and $\boldsymbol{\omega}$ are $g_{\alpha \beta}, \omega^{\alpha}=g^{\alpha \beta} \omega_{\beta}$ and $\omega_{\alpha}=g_{\alpha \beta} \omega^{\beta}$ respectively.

Given a vector $\overrightarrow{\boldsymbol{v}}$ and a tensor $\boldsymbol{T}$ of type $(0, n)(n \geq 1)$, i.e. a $n$-linear form (a linear form for $n=1$, a bilinear form for $n=2$, etc.), we denote by $\overrightarrow{\boldsymbol{v}} \cdot \boldsymbol{T}(\operatorname{resp} . \boldsymbol{T} \cdot \overrightarrow{\boldsymbol{v}})$ the $(n-1)$-linear form obtained by setting the first (resp. last) argument of $\boldsymbol{T}$ to $\overrightarrow{\boldsymbol{v}}$ :

$$
\begin{aligned}
\vec{v} \cdot T & :=T(\vec{v}, \ldots, \ldots,-) \\
T \cdot \vec{v} & :=T\left({ }_{-}, \ldots,{ }_{-}, \vec{v}\right) .
\end{aligned}
$$

Thanks to the above conventions, we may write the scalar product of two vectors $\overrightarrow{\boldsymbol{u}}$ and $\overrightarrow{\boldsymbol{v}}$ as

$$
\boldsymbol{g}(\overrightarrow{\boldsymbol{u}}, \overrightarrow{\boldsymbol{v}})=\overrightarrow{\boldsymbol{u}} \cdot \boldsymbol{v}=\boldsymbol{u} \cdot \overrightarrow{\boldsymbol{v}}
$$


We denote by $\boldsymbol{\nabla}$. the covariant divergence, with contraction taken on the adjacent index. For instance, for a tensor field $\overrightarrow{\boldsymbol{T}}$ of type $(2,0), \boldsymbol{\nabla} \cdot \overrightarrow{\boldsymbol{T}}$ is the vector field defined by

$$
\nabla \cdot \overrightarrow{\boldsymbol{T}}:=\nabla_{\beta} T^{\beta \alpha} \boldsymbol{e}_{\alpha}
$$

where $\left\{\boldsymbol{e}_{\alpha}\right\}$ is the vector basis with respect to which the components $\nabla_{\gamma} T^{\alpha \beta}$ of $\boldsymbol{\nabla} \overrightarrow{\boldsymbol{T}}$ are taken. (Note that the convention for the divergence does not follow the rule for the contraction with a vector: in 2.4a the contraction is performed on the first index.)

We use Greek letters $\alpha, \beta, \gamma, \delta, \ldots$ for abstract and $\mu, \nu, \kappa, \lambda, \ldots$ for concrete spacetime indices. We also use English letters $a, b, c, \ldots$ for abstract and $i, j, k, \ldots$ for concrete spatial indices. We use geometrized HeavisideLorentz units throughout the paper. We use $\nabla_{\alpha}$ or $\partial_{\alpha}$ to denote the (Eulerian) covariant or partial derivative compatible with a curved or flat metric respectively, and $\partial / \partial x^{\alpha}$ to denote the (Lagrangian) partial derivative of a function $f(x, v)$ with respect to $x$ for fixed $v$. We make extensive use of Lie and exterior derivatives: for a pedagogical introduction to using these concepts in relativistic hydrodynamics, the reader is referred to [58, 59]

\section{B. Hamiltonian flows}

It is often thought that continuum systems necessarily require an infinite dimensional manifold for their description, and one often resorts to a classical field-theory approach, based on an action integral over a Lagrangian density in a spacetime 4-volume. This complicates the derivation of conservation laws from symmetries of the action - one of the main reasons for using an action functional in the first place. In many cases, however, the very definition of a perfect fluid allows one to treat each fluid element as an individual particle interacting with other fluid elements through pressure terms (in addition to electromagnetic or gravitational field terms). If the pressure terms are derivable from a potential, then a particlelike action principle can be found. This approach has been utilized by Carter 27] to derive particle-like conservation laws for neutral perfect fluids and for charged poorly conducting fluids. Here, we review Carter's framework and extend it to baroclinic fluids and perfectly conducting magnetofluids.

\section{Lagrangian dynamics}

The results derived in this section will apply to any classical motion obeying a Lagrangian variation principle. That is, for any particular (particle, fluid or magneto-fluid) flow, there exists a Lagrangian function $L(x, v)$ of the spacetime coordinates $x^{\alpha}$ and canonical 4velocity $v^{\alpha}$, evaluated at $x^{\alpha}(\lambda)$ and $v^{\alpha}(\lambda)$ where $\lambda \in \mathbb{R}$ is a canonical time parameter (which need not necessarily coincide with proper time $\tau$ ) in terms of which the (not necessarily unit) vector

$$
v^{\alpha}=\frac{d x^{\alpha}}{d \lambda}
$$

is defined. The equations of the (particle or fluidelement) worldlines $x^{\alpha}(\lambda)$ can be obtained from the action functional

$$
\mathcal{S}=\int_{\lambda_{1}}^{\lambda_{2}} L(x, v) d \lambda
$$

Extremizing the action keeping the endpoints fixed yields the Euler-Lagrange equations of motion

$$
\begin{aligned}
\frac{d p_{\alpha}}{d \lambda} & =\frac{\partial L}{\partial x^{\alpha}} \\
p_{\alpha} & =\frac{\partial L}{\partial v^{\alpha}}
\end{aligned}
$$

where $p_{\alpha}$ is the canonical momentum 1-form conjugate to $x^{\alpha}$. In the context of fluid theory, it is preferable to write the above equations in the (Eulerian) covariant form [27]

$$
£_{\overrightarrow{\boldsymbol{v}}} p_{\alpha}=\nabla_{\alpha} L
$$

or, in exterior calculus notation,

$$
£_{\overrightarrow{\boldsymbol{v}}} \boldsymbol{p}=\mathbf{d} L
$$

where $£_{\overrightarrow{\boldsymbol{v}}}$ is the Lie derivative along the vector $\overrightarrow{\boldsymbol{v}}$ and $\mathbf{d}$ is the exterior derivative 58 60]. The canonical momentum one-form $\boldsymbol{p}=p_{\mu} \mathbf{d} x^{\mu}$ is also known as the tautological one-form, the Liouville one-form, the Poincaré one-form the symplectic potential or simply the canonical one-form 61]. Using the definition of the Lie derivative and the chain rule, the above equation 1 can be expressed as:

$$
\begin{aligned}
£_{\overrightarrow{\boldsymbol{v}}} p_{\alpha}-\nabla_{\alpha} L & :=v^{\beta} \frac{\partial p_{\alpha}}{\partial x^{\beta}}+p_{\beta} \frac{\partial v^{\beta}}{\partial x^{\alpha}}-\left(\frac{\partial L}{\partial x^{\alpha}}+\frac{\partial L}{\partial v^{\beta}} \frac{\partial v^{\beta}}{\partial x^{\alpha}}\right) \\
& =\frac{d p_{\alpha}}{d \lambda}-\frac{\partial L}{\partial x^{\alpha}}+\left(p_{\beta}-\frac{\partial L}{\partial v^{\beta}}\right) \frac{\partial v_{\beta}}{\partial x^{\alpha}} \cdot(2.11)
\end{aligned}
$$

This quantity vanishes iff the Euler-Lagrange equations (2.9) are satisfied; the latter are thus equivalent to the covariant equation (2.10).

\footnotetext{
${ }^{1}$ In Eq. 2.9b), the Lagrangian $L$ and canonical momentum $\boldsymbol{p}$ are regarded functions of the time parameter $\lambda$, through $\overrightarrow{\boldsymbol{x}}(\lambda)$ and $\overrightarrow{\boldsymbol{v}}(\lambda)$, and characterize a single fluid element. In Eq. 2.10), the Lagrangian and canonical momentum are regarded functions on spacetime through $\overrightarrow{\boldsymbol{x}}$ and $\overrightarrow{\boldsymbol{v}}(x)$. They amount to the Lagrangian and canonical momentum of the fluid element located at $\overrightarrow{\boldsymbol{x}}$, and changing the argument $\overrightarrow{\boldsymbol{x}}$ generally changes the fluid element which $L$ and $\boldsymbol{p}$ refer to.
} 


\section{Hamiltonian dynamics}

The Legendre transformation

$$
H=v^{\alpha} p_{\alpha}-L
$$

defines the super-Hamiltonian $H(x, p)$. Then, the equations of motion take the form of Hamilton's equations

$$
\begin{aligned}
\frac{d p_{\alpha}}{d \lambda} & =-\frac{\partial H}{\partial x^{\alpha}} \\
\frac{d x^{\alpha}}{d \lambda} & =\frac{\partial H}{\partial p_{\alpha}}
\end{aligned}
$$

The above equations can be written covariantly as [27]

$$
v^{\beta}\left(\nabla_{\beta} p_{\alpha}-\nabla_{\alpha} p_{\beta}\right)=-\nabla_{\alpha} H
$$

or, in exterior calculus notation,

$$
\vec{v} \cdot \mathbf{d} p=-\mathbf{d} H
$$

One may obtain Eq. (2.14) using the Cartan identity

$$
£_{\overrightarrow{\boldsymbol{v}}} p_{\alpha}=v^{\beta}\left(\nabla_{\beta} p_{\alpha}-\nabla_{\alpha} p_{\beta}\right)+\nabla_{\alpha}\left(v^{\beta} p_{\beta}\right)
$$

or

$$
£_{\vec{v}} p=\vec{v} \cdot \mathrm{d} p+\mathrm{d}(\vec{v} \cdot \boldsymbol{p})
$$

and the Legendre transformation (2.12) to write the covariant Euler-Lagrange equations (2.10) as

$$
£_{\vec{v}} \boldsymbol{p}-\mathbf{d} L=\overrightarrow{\boldsymbol{v}} \cdot \mathbf{d} \boldsymbol{p}+\mathbf{d} H=0 .
$$

Alternatively, one may prove the equivalence of Eq. (2.14) to the Hamilton equations 2.13a) by proceeding analogously to Eq. 2.11), that is, by using the chain rule to rewrite the (Eulerian) covariant derivative $\nabla_{\alpha} H=$ $\partial H / \partial x^{\alpha}+\partial p_{\beta} / \partial x^{\alpha} \partial H / \partial p_{\beta}$ in terms of (Lagrangian) partial derivatives.

\section{Conservation laws}

\section{Poincaré-Cartan integral invariant}

The 2-form

$$
\Omega_{\alpha \beta}:=\nabla_{\beta} p_{\alpha}-\nabla_{\alpha} p_{\beta}
$$

or, equivalently,

$$
\Omega:=\mathbf{d} \boldsymbol{p}=\mathbf{d} p_{\mu} \wedge \mathbf{d} x^{\mu}
$$

is the canonical symplectic form, also known as the Poincaré two-form 61]. Its physical content depends on the action (2.8). In $\S$ III it will be shown that, if the action describes a perfect fluid, then $\Omega$ is Khalatnikov's canonical vorticity tensor; if the action describes a purely magnetic field, then $\boldsymbol{\Omega}$ is the Faraday tensor. Nevertheless, the results of $\S$ apply to any generic Hamiltonian flow; no assumptions on the physical content of the action (2.8) will be made prior to $\S$ III

Taking the exterior derivative of (2.10), commuting the exterior derivative $\mathbf{d}$ with the Lie derivative $£_{\vec{v}}$ and using the identity $\mathbf{d}^{2}=0$, we immediately deduce that the canonical symplectic form 2.17) is advected by the flow:

$$
£_{\overrightarrow{\boldsymbol{v}}} \Omega=0 .
$$

The above equation also follows directly from the Hamilton equations (2.14) and the Cartan identity

$$
£_{\vec{v}} \Omega=\vec{v} \cdot \mathrm{d} \Omega+\mathrm{d}(\overrightarrow{\boldsymbol{v}} \cdot \Omega)
$$

The conservation equation (2.18) is tied to an important integral invariant: Consider ${ }^{2}$ the family $\Psi_{\lambda}$ of diffeomorphisms generated by canonical velocity $\overrightarrow{\boldsymbol{v}}$, with $\Psi_{\lambda}^{-1}$ its inverse. Let $c$ be a ring in the flow, bounding a 2surface $S$; let $c_{\lambda}=\Psi_{\lambda}(c)$ be the family of rings dragged along by the flow, bounding the 2 -surfaces $S_{\lambda}=\Psi_{\lambda}(S)$. That is, each point of $S_{\lambda}$ is obtained by moving each point of $S$ an affine time $\lambda$ along the flow through that point. The closed line integral of $\boldsymbol{p}$ around $\mathcal{C}_{\lambda}$ can then be written as

$$
\mathcal{I}:=\oint_{c_{\lambda}} \boldsymbol{p}=\int_{S_{\lambda}} \boldsymbol{\Omega}=\int_{S} \Psi_{\lambda}^{-1} \boldsymbol{\Omega}
$$

where we used the Stokes theorem (relating the circulation integral $\oint_{c_{\lambda}} p_{\alpha} d x^{\alpha}$ with the integral $\int_{S_{\lambda}} \Omega_{\alpha \beta} d x_{(1)}^{\alpha} d x_{(2)}^{\beta}$ of $\boldsymbol{\Omega}$ on $S_{\lambda}$, where $d x_{(1)}^{\alpha}$ and $d x_{(2)}^{\beta}$ are infinitesimal vectors tangent to $S_{\lambda}$ spanning the tangent space at each point) and the diffeomorphism invariance of an integral (i.e. the identity $\int_{\Psi_{\lambda}(S)} \Psi_{\lambda} \boldsymbol{\Omega}=\int_{S} \boldsymbol{\Omega}$, with $\Omega$ replaced by $\Psi_{\lambda}^{-1} \Omega$, cf. Eq. (A.81) in [60]). Eq. (2.18) implies that the above integral is conserved:

$$
\frac{d \mathcal{I}}{d \lambda}=\int_{S} \frac{d}{d \lambda}\left(\Psi_{\lambda}^{-1} \boldsymbol{\Omega}\right)=\int_{S} £_{\vec{v}} \boldsymbol{\Omega}=0 .
$$

The closed line integral (2.20) is known in analytical dynamics as the Poincaré-Cartan integral invariant associated with Hamiltonian systems 62 64. Its existence emerges from the Hamiltonian structure of Eq. (2.14). In particular, a dynamical system possesses a PoincaréCartan integral invariant if and only if it is Hamiltonian [65.

Although this result is well-known in analytical dynamics, to our knowledge, its applicability to (magneto)hydrodynamics was only recognized by Carter [27] Some classical mechanics texts mention that the integral

\footnotetext{
2 This derivation follows and generalizes Friedman \& Stergioulas' 60] proof of conservation of circulation.
} 
(2.20) corresponds to a conserved circulation in phase space, analogous to Kelvin's circulation integral in a barotropic fluid. In fact, this is more than a mere analogy, albeit in the converse direction: Kelvin's circulation equals the integral (2.20) if the Lagrangian is chosen to be that of a perfect barotropic fluid element, Eq. (3.20) [27, 66]. Similarly, Alfvén's magnetic flux theorem, and the generalizations of Kelvin's theorem to poorly [27] or perfectly conducting [29 31] magnetofluids, emerge also as special cases of the Poincaré-Cartan integral invariant (2.20). This will be shown in $\S$ III by constructing the appropriate Lagrangians.

\section{Irrotational flows}

In general, a flow will be called irrotational iff the canonical vorticity 2 -form vanishes:

$$
\Omega=\mathbf{d} p=0 .
$$

Then, if the domain $\mathcal{D}$ is simply connected, the Poincaré lemma implies the local existence of a single-valued scalar field $\mathcal{S}$ such that

$$
p=\mathrm{d} \mathcal{S}
$$

or, equivalently,

$$
p_{\alpha}=\nabla_{\alpha} \mathcal{S}
$$

The invariance of the Poincaré-Cartan integral (2.20) guarantees that initially irrotational flows remain irrotationa 3 . This is very useful when solving the Cauchy problem with irrotational initial data (cf. [66] for a $3+1$ evolution scheme exploiting this property in barotropic fluids). For an irrotational flow, substituting Eq. (2.23) into the equations of motion (2.10), (2.14), we find that the latter have first integrals:

$$
\begin{aligned}
£_{\overrightarrow{\boldsymbol{v}}} \mathcal{S}-L & =0 \\
H & =0
\end{aligned}
$$

respectively. In general, a system with constant $H$ is called uniformly canonical. This is the case for irrotational flow, and, more generally, for a perfect fluid that is homentropic or barotropic, as will be shown below.

We note that the above first integrals hold throughout the flow. Indeed, taking the exterior derivative of the above equations and commuting the operator $\mathbf{d}$ with $£_{\boldsymbol{v}}$ leads back to the equations of motion (2.10), (2.14). In the above integrals, we have dropped an additive integration constant by absorbing it into the definition of the potential $\mathcal{S}$. Note that Eq. (2.24) follows directly from

\footnotetext{
${ }^{3}$ In the context of barotropic fluids, this is known as Helmholtz's third theorem, which is a corollary of Kelvin's circulation theorem.
}

Eq. (2.25) with the aid of Eqs. (2.12) and (2.23) and the definition of the Lie derivative.

Eqs. (2.24) and (2.25) were derived for an irrotational flow. More generally, the same equations can be shown to hold for helicity-free flows which are representable in the Clebsch form $\boldsymbol{p}=\mathbf{d} \mathcal{S}+\alpha \mathbf{d} \beta$. This follows by substituting the latter expression into the equations of motion (2.10), (2.14) and using the fact that the Clebsch potentials $\alpha, \beta$ are advected by the flow, that is $£_{\overrightarrow{\boldsymbol{v}}} \alpha=0, £_{\overrightarrow{\boldsymbol{v}}} \beta=0$.

\section{Poincaré two-form}

Let $u^{\alpha}=v^{\alpha}\left(-v^{\beta} v_{\beta}\right)^{-1 / 2}$ be the unit vector along $v^{\alpha}$. In light of Eq. (2.14), we can decompose the 2-form (2.17) into 'electric' and 'magnetic' parts with respect to $u^{\alpha}$ as

$$
\Omega_{\alpha \beta}=\left(-v^{\beta} v_{\beta}\right)^{-1 / 2}\left(u_{\alpha} \nabla_{\beta} H-u_{\beta} \nabla_{\alpha} H\right)+u^{\delta} \epsilon_{\delta \alpha \beta \gamma} \omega^{\gamma}
$$

or

$$
\begin{aligned}
\boldsymbol{\Omega} & =(-\overrightarrow{\boldsymbol{v}} \cdot \boldsymbol{v})^{-1 / 2} \boldsymbol{u} \wedge \mathrm{d} H+\star(\boldsymbol{u} \wedge \boldsymbol{\omega}) \\
\star \boldsymbol{\Omega} & =(-\overrightarrow{\boldsymbol{v}} \cdot \boldsymbol{v})^{-1 / 2} \star(\boldsymbol{u} \wedge \mathrm{d} H)-\boldsymbol{u} \wedge \boldsymbol{\omega},
\end{aligned}
$$

where

$$
\omega_{\alpha}:=\frac{1}{2} u^{\delta} \epsilon_{\delta \alpha \beta \gamma} \Omega^{\beta \gamma}=u^{\delta} \star \Omega_{\delta \alpha}
$$

or

$$
\omega:=\vec{u} \cdot \star \Omega
$$

From the antisymmetry properties of $\epsilon$ it follows that

$$
\overrightarrow{\boldsymbol{u}} \cdot \mathbf{d} H=0, \quad \overrightarrow{\boldsymbol{u}} \cdot \boldsymbol{\omega}=0
$$

and that the scalar invariants of the 2 -form $\Omega$ are

$$
\begin{aligned}
\frac{1}{2} \Omega^{\alpha \beta} \Omega_{\alpha \beta} & =\omega^{\alpha} \omega_{\alpha}-\left(-v^{\beta} v_{\beta}\right)^{-1} \nabla^{\alpha} H \nabla_{\alpha} H \\
\frac{1}{2}\left(\star \Omega^{\alpha \beta}\right) \Omega_{\alpha \beta} & =\left(-v^{\beta} v_{\beta}\right)^{-1 / 2} \omega^{\alpha} \nabla_{\alpha} H
\end{aligned}
$$

By the definition (2.17), $\Omega$ is an exact 2-form. Because $\mathbf{d}^{\mathbf{2}}=\mathbf{0}$, any exact 2 -form is also closed:

$$
\mathbf{d} \Omega=0 \Leftrightarrow \nabla_{\alpha}\left(\star \Omega^{\alpha \beta}\right)=0
$$

Given a scalar field $\phi(x)$ on $\mathscr{M}$, one can construct an exact 1-form

$$
l=\mathbf{d} \phi
$$

which is also, by virtue of the identity $\mathbf{d}^{\mathbf{2}}=\mathbf{0}$, closed:

$$
\mathbf{d} \boldsymbol{l}=0 \Leftrightarrow \nabla_{\alpha} l_{\beta}-\nabla_{\beta} l_{\alpha}=0 .
$$

Given a closed 2 -form $\boldsymbol{\Omega}$ and a closed 1 -form $\boldsymbol{l}$, one can construct a current $j^{\alpha}:=l_{\beta} \star \Omega^{\beta \alpha}$, or

$$
j:=\vec{l} \cdot \star \Omega .
$$


which, by virtue of Eqs. (2.31) and (2.33), is conserved:

$$
\nabla_{\alpha} j^{\alpha}=\nabla_{\alpha}\left(\nabla_{\beta} \phi \star \Omega^{\beta \alpha}\right)=0 .
$$

This conservation law implies a corresponding global conservation of the integrated flux of $j^{\alpha}$ across a hypersurface.

An infinite number of (not necessarily independent) conservation laws stem from Eq. (3.49) since, in general, $\phi(x)$ can be any differentiable function of the coordinates. For example, in a chart $\left\{x^{\mu}\right\}=\left\{t, x^{i}\right\}$, if $\phi$ is chosen to be the spatial coordinate $x^{1}$, the above equation reduces to the $x^{1}$-component of Eq. (2.31). If $\phi$ coincides with coordinate time $t$, Eq. (3.49) yields a spatial constraint equation. Other combinations of the coordinates give different projections Eq. (2.31). Choosing $\phi$ to be the super-Hamiltonian $H$ gives rise to a conserved current

$$
j^{\alpha}:=\nabla_{\beta} H \star \Omega^{\beta \alpha}
$$

This conservation law holds for any Hamiltonian system with a Poincaré 2 -form $\Omega$. For a baroclinic fluid, described by Eq. (3.41) below, the time component of this current is the potential vorticity, as shown in $\S$ III The corresponding conservation law, known as Ertel's theorem [67, 68], arises simply as a special case of Eq. (2.36).

As mentioned earlier, a system with spatially constant super-Hamiltonian $H$ is uniformly canonical. If the uniformity condition $\mathbf{d} H=0$ holds on an initial hypersurface, then Eq. (2.28) guarantees that the condition is preserved in time. For such systems, Eqs. (2.26a) and (2.31) yield the conservation law

$$
\mathbf{d} \star(\boldsymbol{u} \wedge \boldsymbol{\omega})=0 \Leftrightarrow \nabla_{\alpha}\left(u^{\alpha} \omega^{\beta}-u^{\beta} \omega^{\alpha}\right)=0
$$

In $3+1$ dimensions, this equation is the curl of Eq. (2.18). Helmholtz's vorticity transport equation 68] and Alfven's magnetic field transport equation [69] are special cases of this general conservation law.

\section{Generalized Helicity}

Eqs. (2.17), (2.31) and (2.30) imply that, for uniformly canonical systems, the generalized helicity current

$$
h:=\vec{p} \cdot \star \Omega
$$

is conserved:

$$
\nabla_{\alpha} h^{\alpha}=\frac{1}{2} \Omega_{\alpha \beta} \star \Omega^{\beta \alpha}=\omega^{\alpha} \nabla_{\alpha} H=0 .
$$

This conservation law also implies a corresponding global conservation of the integrated flux of $h^{\alpha}$ across a hypersurface. Specific examples are given in $\S$ Џ1I 70 72].

\section{Noether's theorem}

Noether's theorem states that each continuous symmetry of the action implies a quantity conserved by the motion. In particular, the generalized Noether theorem may be stated as follows [73]. Consider the $\varepsilon$-family of infinitesimal coordinate transformations

$$
\overrightarrow{\boldsymbol{x}} \rightarrow \overrightarrow{\boldsymbol{x}}_{\varepsilon}=\overrightarrow{\boldsymbol{x}}+\varepsilon \overrightarrow{\boldsymbol{k}}(x, v)
$$

generated by the vector field $\overrightarrow{\boldsymbol{k}}(x, v)$, which can depend on position and velocity, for a small parameter $\varepsilon$. If these transformations leave the action (2.8) unchanged or, equivalently, change the Lagrangian $L(x, v)$ by a total derivative of some scalar $K(x)$,

$$
L \rightarrow L_{\varepsilon}=L-\varepsilon \frac{d K}{d \lambda},
$$

then the quantity

$$
\mathcal{C}(x, v)=\frac{\partial L}{\partial v^{\alpha}} k^{\alpha}+K
$$

is a constant of motion:

$$
\frac{d \mathcal{C}}{d \lambda}=£_{\overrightarrow{\boldsymbol{v}}} \mathcal{C}=0
$$

If $\overrightarrow{\boldsymbol{k}}$ depends on velocity, then the family (2.40) of transformations is not generally considered a family of diffeomorphisms. It is, however, a generalized symmetry of the action and Noether-related to an invariant of the form (2.42).

Conversely, the inverse Noether theorem [73] may be stated as follows: if the quantity $\mathcal{C}(x, v)$ is a constant of motion, then the $\varepsilon$-family of infinitesimal transformations generated by the vector field $\overrightarrow{\boldsymbol{k}}(x, v)$, obtained by solving the linear system

$$
\frac{\partial^{2} L}{\partial v^{\alpha} \partial v^{\beta}} k^{\beta}=\frac{\partial \mathcal{C}}{\partial v^{\alpha}},
$$

is a generalized symmetry of the action.

In the Hamiltonian picture, a scalar quantity $\mathcal{C}(x, p)$, which does not explicitly depend on the time parameter $\lambda$, is conserved if it commutes with the superHamiltonian, in the sense of a vanishing Poisson bracket:

$$
\frac{d \mathcal{C}}{d \lambda}=£_{\overrightarrow{\boldsymbol{v}}} \mathcal{C}=\{\mathcal{C}, H\} \equiv \frac{\partial \mathcal{C}}{\partial x^{\gamma}} \frac{\partial H}{\partial p_{\gamma}}-\frac{\partial \mathcal{C}}{\partial p_{\gamma}} \frac{\partial H}{\partial x^{\gamma}}=0 .
$$

Conserved quantities polynomial in the momenta are associated with Killing vectors or tensors and are Noetherrelated to symmetries of the action, as discussed below. The super-Hamiltonian $H$ does not explicitly depend on the affine parameter $\lambda$ and is itself a constant of motion, in agreement with Eq. (2.28) (this symmetry is Noetherrelated to the metric tensor being a Killing tensor, as discussed in $\S$ 【I).

For barotropic fluids, Eqs. (2.42) and (2.43) or (2.45) give rise to Bernoulli's law, as shown in the next section. 


\section{EXAMPLES OF HAMILTONIAN FLOWS}

\section{A. Perfect fluids}

We assume that a part $\mathcal{D} \subset \mathscr{M}$ of spacetime is occupied by a perfect fluid, characterized by the energymomentum tensor

$$
\boldsymbol{T}^{\mathrm{fl}}=(\epsilon+p) \boldsymbol{u} \otimes \boldsymbol{u}+p \boldsymbol{g},
$$

where $\epsilon$ is the proper energy density, $p$ is the fluid pressure and $u^{\alpha}=d x^{\alpha} / d \tau$ is the fluid 4-velocity. Moreover, we neglect effects of viscosity or heat conduction and we assume that the fluid is a simple fluid, that is, all thermodynamic quantities depend only on the entropy density $s$ and proper baryon number density $n$. In particular,

$$
\epsilon=\epsilon(s, n) \text {. }
$$

The above relation is called the equation of state (EOS) of the fluid. The temperature $T$ and the baryon chemical potential $\mu$ are then defined by

$$
T:=\frac{\partial \epsilon}{\partial s} \quad \text { and } \quad \mu:=\frac{\partial \epsilon}{\partial n} .
$$

Then, the first law of thermodynamics can be written as

$$
d \epsilon=\mu d n+T d s
$$

As a consequence, $p$ is a function of $(s, n)$ entirely determined by (3.2):

$$
p=-\epsilon+T s+\mu n .
$$

Let us introduce the specific enthalpy,

$$
h:=\frac{\epsilon+p}{\rho}=g+T S,
$$

where $\rho$ is the rest-mass density

$$
\rho:=m n
$$

$g$ is the specific Gibbs free energy

$$
g:=\frac{\mu}{m}
$$

$m=1.66 \times 10^{-27} \mathrm{~kg}$ is the baryon rest-mass, and $S$ is the specific entropy, or entropy per particle:

$$
S:=\frac{s}{\rho}
$$

The second equality in (3.6) is an immediate consequence of (3.5). From Eqs. (3.4)-3.9), we obtain the thermodynamic relations

$$
d \epsilon=h d \rho+\rho T d S, \quad d p=\rho(d h-T d S)
$$

A simple perfect fluid is barotropic if the energy density depends only on the pressure, $\epsilon=\epsilon(p)$. This is the case for a cold or a homentropic fluid.
With the aid of Eqs. (3.4 - 3.10), the divergence of the fluid energy-momentum tensor (3.1) can be decomposed as

$$
\vec{\nabla} \cdot \boldsymbol{T}^{\mathrm{fl}}=h \boldsymbol{u}[\boldsymbol{\nabla} \cdot(\rho \overrightarrow{\boldsymbol{u}})]+\rho[\overrightarrow{\boldsymbol{u}} \cdot \mathbf{d}(h \boldsymbol{u})-T \mathbf{d} S] .
$$

Conservation of rest mass

$$
\nabla \cdot(\rho \overrightarrow{\boldsymbol{u}})=0,
$$

and the vanishing of 3.11 yield the relativistic Euler equation for baroclinic fluids, in the canonical form:

$$
£_{\overrightarrow{\boldsymbol{u}}}(h \boldsymbol{u})+\mathbf{d} h=\overrightarrow{\boldsymbol{u}} \cdot \mathbf{d}(h \boldsymbol{u})=T \mathbf{d} S
$$

where the first equality follows from the Cartan identity (2.15) and the normalization condition

$$
g_{\alpha \beta} u^{\alpha} u^{\beta}=-1 .
$$

For barotropic fluids, the Euler equation (3.13) simplifies to

$$
£_{\vec{u}}(h \boldsymbol{u})+\mathbf{d} h=\overrightarrow{\boldsymbol{u}} \cdot \mathbf{d}(h \boldsymbol{u})=0 .
$$

Eq. 3.15 was obtained in special relativity by Synge (1937) 17] and in general relativity by Lichnerowicz (1941) [18]. The extension (3.13) to baroclinic (nonisentropic) fluids was obtained by Taub (1959) [74] (see also [27, 58, 75]). Both of these relativistic hydrodynamic equations are canonical and can be described within the framework of $\S$ II which provides a very efficient approach to the derivation of conservation laws.

\section{B. Barotropic flows}

\section{Hamilton's principle for a barotropic-fluid element}

The Euler equation (3.15) for a barotropic fluid is readily in the canonical form (2.14). Thus, a particle variational principle in the form described in $\S \prod$ can be found. Indeed the motions of fluid elements in a barotropic fluid are conformally geodesic, that is, they are geodesics of a manifold with metric $h^{2} g_{\alpha \beta}[17,[18,76]$. This follows from the fact that Eq. 3.15) is the Euler-Lagrange equation of the action functional

$$
\mathcal{S}=-\int_{\tau_{1}}^{\tau_{2}} h d \tau=-\int_{\tau_{1}}^{\tau_{2}} h \sqrt{-g_{\alpha \beta} \frac{d x^{\alpha}}{d \tau} \frac{d x^{\beta}}{d \tau}} d \tau .
$$

The Lagrangian

$$
L(x, u)=-h \sqrt{-g_{\alpha \beta} u^{\alpha} u^{\beta}}
$$

is associated with the canonical momentum 1 -form

$$
\boldsymbol{p}=h \boldsymbol{u},
$$

and the canonical vorticity 2 -form

$$
\boldsymbol{\Omega}=\mathbf{d}(h \boldsymbol{u}) .
$$


On-shell, the condition (3.14) is satisfied, and the Lagrangian (3.17) takes the value $L=-h$. Carter 27] introduced a slightly modified Lagrangian

$$
L(x, u)=\frac{1}{2} h g_{\alpha \beta} u^{\alpha} u^{\beta}-\frac{1}{2} h,
$$

that is associated with the same equations of motion and has the same on-shell value, but its action is not reparametrization invariant. Thus, if one wishes, for instance, to use reparametrization invariance to replace proper time $\tau$ by coordinate time $t$, in order to obtain a constrained Hamiltonian via $3+1$ decomposition, as done in [66], then the action (3.16) is the appropriate starting point. If, on the other hand, one is interested in a super-Hamiltonian that describes the dynamics in a 4dimensional spacetime, then Carter's Lagrangian (3.20) is more suitable. Substituting the latter into the Legendre transformation (2.12) yields the super-Hamiltonian

$$
H(x, p)=\frac{1}{2 h} g^{\alpha \beta} p_{\alpha} p_{\beta}+\frac{1}{2} h
$$

which vanishes on-shell (whence Eq. (3.14) holds). Substituting Eqs. (3.18) and (3.21) into the Hamilton equation (2.14) yields the barotropic Euler equation (3.15).

\section{Conservation of circulation in barotropic flows}

For this system, Eq. (2.18) yields a relativistic generalization of Helmholtz's vorticity conservation equation:

$$
£_{\overrightarrow{\boldsymbol{u}}} \mathbf{d}(h \boldsymbol{u})=0
$$

and the Poincaré-Cartan integral invariant (2.20)-2.21) gives rise a relativistic generalization of Kelvin circulation theorem: the circulation along a fluid ring $c_{\tau}$ dragged along by the flow is conserved:

$$
\frac{d}{d \tau} \oint_{c_{\tau}} h \boldsymbol{u}=0
$$

Conservation of circulation for the nonrelativistic Euler equations was discovered by Cauchy (1815) [77, 78] and independently rediscovered by Kelvin (1869) 79]. The extension of this theorem to relativistic barotropic fluids was obtained by Lichnerowicz and [80] Taub [4]. The most interesting feature of the above conservation law is that its derivation does not depend on the spacetime metric or spacetime symmetries. Thus, it is exact in time-dependent spacetimes, with gravitational waves carrying energy and angular momentum away from a system. Oscillating stars and radiating binaries, if modeled as barotropic fluids with no viscosity or dissipation other than gravitational waves, exactly conserve circulation [60].

\section{Fluid helicity}

Since the super-Hamiltonian (3.21) is constant, the system is uniformly canonical, and helicity is conserved. If we we substitute Eq. (3.18) into Eq. (2.38), then Eqs. (2.27) and (2.39) imply that the fluid helicity current $[27,81,82]$

$$
\boldsymbol{h}_{\mathrm{fl}}:=h \overrightarrow{\boldsymbol{u}} \cdot \star \boldsymbol{\Omega}=h \boldsymbol{\omega}
$$

is conserved:

$$
\nabla_{\alpha}\left(h u_{\beta} \star \Omega^{\beta \alpha}\right)=\nabla_{\beta}\left(h \omega^{\beta}\right)=0 .
$$

This implies a corresponding global conservation of the integrated flux of $h_{\mathrm{em}}^{\alpha}$ across a spatial hypersurface. In a chart $\left\{t, x^{i}\right\}$, the volume integral of the time component of $\overrightarrow{\boldsymbol{h}}_{\mathrm{fl}}$ :

$$
h_{\mathrm{fl}}^{t}:=\overrightarrow{\boldsymbol{h}}_{\mathrm{fl}} \cdot \nabla t=h \omega^{t}=h u_{i} \star \Omega^{i t}=-h \omega^{i} u_{i} / u_{t}
$$

is the relativistic generalization of Moffat's fluid helicity [32, 71, 72]. The last equality follows from Eq. (2.28). If the vorticity $\omega^{i}$ has sufficient decay, then the total volume integral of the above quantity is conserved by the flow.

\section{Killing vector fields \& Bernoulli's law}

If there exists a vector field $k^{\alpha}(x)$, that generates a family of diffeomorphisms (2.40) leaving the Langrangian (3.17) unchanged, then Noether's theorem implies the existence of a streamline invariant linear in the momenta, given by Eq. (2.42) (with $K$ set to zero):

$$
\mathcal{E}(x, p)=k^{\alpha} p_{\alpha}=h u_{\alpha} k^{\alpha} .
$$

As stated by Eq. (2.43), this quantity is conserved along a streamline (i.e. the trajectory of a fluid element):

$$
\frac{d \mathcal{E}}{d \tau}=£_{\overrightarrow{\boldsymbol{u}}} \mathcal{E}=\overrightarrow{\boldsymbol{u}} \cdot \nabla \mathcal{E}=0 .
$$

The above statement is a generalization of Bernoulli's law to relativistic barotropic fluids. In light of the above, each Bernoulli-type conservation law is Noether-related to a continuous symmetry of the flow.

Given the super-Hamiltonian (3.21), one may directly verify when a quantity of the form (3.27) is conserved by computing the Poisson bracket (2.45):

$$
\begin{aligned}
\frac{d \mathcal{E}}{d \tau} & =\{\mathcal{E}, H\}=\frac{1}{h} p_{\alpha} p_{\beta} \nabla^{\alpha} k^{\beta}-k^{\gamma} \nabla_{\gamma} h \\
& =\frac{1}{2 h} u^{\alpha} u^{\beta} £_{\overrightarrow{\boldsymbol{k}}}\left(h^{2} g_{\alpha \beta}\right)
\end{aligned}
$$

which vanishes for all timelike streamlines iff

$$
£_{\overrightarrow{\boldsymbol{k}}}\left(h^{2} \boldsymbol{g}\right)=0 .
$$

That is, the necessary and sufficient condition for $\mathcal{E}$ to be a streamline invariant is that $\overrightarrow{\boldsymbol{k}}$ be a Killing vector of a 
manifold with metric $h^{2} \boldsymbol{g}$. This result is intuitive given the fact that, as mentioned earlier, the fluid streamlines are geodesics of this conformal metric, cf. Eq. (3.16). We remark that the vanishing of both $£_{\vec{k}} \boldsymbol{g}$ and $£_{\vec{k}} h$, as indicated by the first line of Eq. (3.29), is a sufficient but not necessary condition for $\mathcal{E}$ to be conserved.

When the pressure vanishes, i.e. when $h=1$, the condition (3.30) reduces to the Killing equation $\nabla_{(\alpha} k_{\beta)}=$ 0 , which is Noether-related to the existence of conserved quantities linear in the momenta for geodesic motion 83 85.).

As an example, let us consider a helically symmetric, rigidly rotating fluid equilibrium, such as a rigidly rotating star (that may be triaxially deformed [86]), or a tidally-locked binary on circular orbits. The flow field may then be written as

$$
\overrightarrow{\boldsymbol{u}}=u^{t} \overrightarrow{\boldsymbol{k}}
$$

where

$$
\vec{k}=\vec{t}+\Omega \vec{\varphi}
$$

is a helical Killing vector field which Lie-derives the metric: $£_{\vec{k}} \boldsymbol{g}=0$. Here, $\Omega$ is the rotation frequency, $\overrightarrow{\boldsymbol{t}}=\partial_{t}$ is the generator of time translations and $\vec{\varphi}=\partial_{\varphi}$ is the generator of rotations about the rotation axis.

Let us assume that the fluid configuration is helically symmetric, that is, the Lie derivatives of all fluid variables (such as $\rho, h, \boldsymbol{u}$ ) along $\overrightarrow{\boldsymbol{k}}$ vanish. Since, by virtue of Eq. (3.30), the system is stationary in a rotating frame, Noether's theorem guarantees that the energy in a rotating frame, given by Eq. (3.27):

$$
\mathcal{E}=k^{\alpha} p_{\alpha}=p_{t}+\Omega p_{\varphi}
$$

is conserved along streamlines.

In general, this quantity can differ from one streamline to the next. However, a stronger result follows from Eq. (3.31) and the Cartan identity (2.15), which allow one to write the Euler equation (3.15) as

$$
\overrightarrow{\boldsymbol{k}} \cdot \mathbf{d} \boldsymbol{p}=£_{\overrightarrow{\boldsymbol{k}}} \boldsymbol{p}-\mathbf{d}(\overrightarrow{\boldsymbol{k}} \cdot \boldsymbol{p})=0 .
$$

Because $£_{\vec{k}} \boldsymbol{p}=0$, the first integral (3.27) of the Euler equation is constant throughout the fluid:

$$
\nabla \mathcal{E}=0 .
$$

This stronger conservation law is a relativistic generalization of von Zeipel's law 60]. The energy (3.27) is also a first integral to the Euler equation if a helically symmetric system is irrotational [57, 87 94]. Such first integrals are valuable for solving for obtaining fluid equilibria via the self-consistent field method [95]. Generalizations of these first integrals have been used to construct equilibria for spinning [96 99] or eccentric [100, 101] compact binaries in numerical relativity.

\section{Killing tensor fields $\&$ the Carter constant}

Geodesic motion of test particles in Kerr (or Kerr-de Sitter) spacetimes is known to admit a fourth constant of motion (in addition to energy, angular momentum, and four-velocity magnitude), known as the Carter constant, which is quadratic in the momenta and is Noether-related to the existence of a Killing tensor field [83].

To our knowledge, the concept of a Killing tensor for fluid flows has not been defined before, but the framework outlined $\S \amalg$ provides the means to do so. Consider a tensor field $K^{\alpha \beta}(x)$ associated with a streamline invariant quadratic in the momenta,

$$
\mathcal{E}=K^{\alpha \beta} p_{\alpha} p_{\beta}+K,
$$

where the scalar $K(x)$ is a function of position. This invariant can be considered a special case of the invariant (2.42) and follows from the generalized Noether theorem, with $k^{\alpha}(x, p)=K^{\alpha \beta}(x) p_{\beta}$ being the generator of the symmetry transformations 102. For the barotropic fluid super-Hamiltonian (3.21), the Poisson bracket (2.45) is

$$
\{\mathcal{E}, H\}=\left(h^{2} \nabla_{\gamma} K_{\alpha \beta}+2 h g_{\alpha \beta} K_{\gamma \delta} \nabla^{\delta} h-g^{\alpha \beta} \nabla^{\gamma} K\right) u^{\alpha} u^{\beta} u^{\gamma} .
$$

The above bracket vanishes for all timelike streamlines iff

$$
h^{2} \nabla_{(\gamma} K_{\alpha \beta)}+2 h g_{(\alpha \beta} K_{\gamma) \delta} \nabla^{\delta} h-g_{(\alpha \beta} \nabla_{\gamma)} K=0
$$

That is, the quantity (3.36) is conserved along streamlines iff $\boldsymbol{K}$ is a Killing tensor of the conformal metric $h^{2} \boldsymbol{g}$.

In the case of a reducible Killing tensor of the form $K^{\alpha \beta}=k^{\alpha} k^{\beta}$, where $k^{\alpha}$ is a Killing vector satisfying Eq. (3.30), the condition (3.38) is automatically satisfied while $K$ again vanishes.

When the pressure vanishes, $h=1$, the scalar $K$ must vanish and the above condition reduces to the Killing equation $\nabla_{(\alpha} K_{\beta \gamma)}=0$, which is the necessary and sufficient condition $K^{\alpha \beta} p_{\alpha} p_{\beta}$ being conserved along a geodesic of $g_{\alpha \beta}$. This is the condition satisfied by the Killing tensor in the Kerr spacetime, which is Noetherrelated to the Carter constant [83, 84]. In light of this, Eqs. (3.36)-(3.38) generalize the concept of a Carter constant to test fluids in Kerr spacetime. Note, however, that the fluid configuration must satisfy a generalized symmetry (in particular, the Hamilton-Jacobi equation describing the flow [66] must be separable in BoyerLindquist coordinates) in order for this constant to exist.

A geodesic flow can be described by the superHamiltonian $H=\frac{1}{2} g^{\alpha \beta} p_{\alpha} p_{\beta}$, with $p_{\alpha}=u_{\alpha}$, which is conserved by virtue of the normalization condition (3.14). This conserved quantity arises from $g^{\alpha \beta}$ being covariantly constant and thus a Killing tensor, and is Noether-related to the super-Hamiltonian being independent of the affine parameter $\tau$. For barotropic flow, however, $g^{\alpha \beta}$ is not a Killing tensor, as it does not satisfy the condition (3.38) except in the geodesic limit. (If $g^{\alpha \beta}$ were a Killing tensor, 
then $g^{\alpha \beta} p_{\alpha} p_{\beta}=-h^{2}$ would be a streamline constant, but this is not true unless $h=1$ ). However, $K^{\alpha \beta}=g^{\alpha \beta} / h$ is a Killing tensor, since it satisfies the condition (3.38) provided that $K=h$. The quadratic streamline constant (3.36) associated with this Killing tensor is simply the super-Hamiltonian (3.21).

\section{Baroclinic flows}

\section{Hamilton's principle for a baroclinic-fluid element}

The possibility of expressing the equations of baroclinic (non-isentropic) fluid flows in canonical form has been demonstrated by Carter [27]. An intuitively simple action principle (different from but equivalent to Carter's) may be obtained as follows.

A free test particle of rest mass $m$, moving along a geodesic of spacetime, extremizes the action $\mathcal{S}=$ $-m \int_{\tau_{1}}^{\tau_{2}} d \tau$ [103. For barotropic flows, as indicated by Eq. (3.16), the pressure force on a fluid element can be accounted for by replacing rest mass by the specific enthalpy $h \mathrm{~m}$. For baroclinic flows, in light of Eqs. (3.6), (3.8) and (3.10), the natural generalization is to replace rest mass in the above action by the chemical potential $\mu=g m$ or, equivalently, the specific Gibbs free energy $g$ (the rest mass can be dropped without affecting the equations of motion). Upon inspection, it becomes immediately clear that Eq. (3.13) is indeed the Euler-Lagrange equation of the action functional

$$
\mathcal{S}=-\int_{\tau_{1}}^{\tau_{2}} g d \tau=-\int_{\lambda_{1}}^{\lambda_{2}}\left(h \sqrt{-g_{\alpha \beta} \frac{d x^{\alpha}}{d \lambda} \frac{d x^{\beta}}{d \lambda}}-S\right) d \lambda
$$

provided that the (non-affine) canonical time parameter

$$
\lambda(\tau):=\int^{\tau} T\left(\tau^{\prime}\right) d \tau^{\prime}
$$

is used to parametrize the action. Note that entropy breaks time-parametrization invariance: unlike the barotropic fluid action (3.16), the baroclinic fluid action (3.39) is not parametrization invariant. Consequently, parameter choices other than (3.40), such as proper time $\tau$ or coordinate time $t$, lead to incorrect equations of motion. The Lagrangian

$$
L(x, v)=-h \sqrt{-g_{\alpha \beta} v^{\alpha} v^{\beta}}+S
$$

is associated, by virtue of Eqs. (2.7) and (2.9b), with the canonical velocity and canonical momentum

$$
\begin{aligned}
& v^{\alpha}=\frac{d x^{\alpha}}{d \lambda}=\frac{1}{T} \frac{d x^{\alpha}}{d \tau}=\frac{1}{T} u^{\alpha} \\
& p_{\alpha}=\frac{\partial L}{\partial v^{\alpha}}=T h v_{\alpha}=h u_{\alpha} .
\end{aligned}
$$

On-shell, by virtue of Eq. (3.14), one has $v^{\alpha} v_{\alpha}=-T^{-2}$ and the Lagrangian takes the value $L=-g / T=-h / T+$
$S$ and, by virtue of Eq. (2.12), the super-Hamiltonian takes the value $H=-S$. Then, the Euler-Lagrange equation (2.10) becomes

$$
£_{\overrightarrow{\boldsymbol{u}} / T}(h \boldsymbol{u})=\mathbf{d}(S-h / T)
$$

and the Hamilton equation (2.14) becomes

$$
\frac{\vec{u}}{T} \cdot \mathbf{d}(h \boldsymbol{u})=\mathbf{d} S .
$$

Both of these equations are equivalent expressions of the relativistic Euler equation (3.13) for baroclinic fluids.

Carter [27] introduced a different Lagrangian analogous to Eq. (3.20)

$$
L(x, v)=\frac{1}{2} T h g_{\alpha \beta} v^{\alpha} v^{\beta}-\frac{1}{2}\left(\frac{g}{T}-S\right),
$$

that is associated with the same canonical velocity and momentum (3.42), has the same on-shell value as our Lagrangian (3.41), and leads to the same equation of motion (3.43). The Legendre transformation (2.12) yields the super-Hamiltonian

$$
H(x, p)=\frac{1}{2 T h} g^{\alpha \beta} p_{\alpha} p_{\beta}+\frac{h}{2 T}-S,
$$

which has the same on-shell value and leads to the canonical equation of motion (3.44).

\section{Conservation of circulation in baroclinic flows}

The canonical momentum and canonical vorticity are given by the same expressions (3.18) and (3.19) as for barotropic flows. However, the vorticity is no longer Liedragged by the fluid four-velocity $\overrightarrow{\boldsymbol{u}}$ : the exterior derivative of Eq. 3.13 reads

$$
£_{\vec{u}} \mathbf{d}(h \boldsymbol{u})=\mathbf{d} T \wedge \mathbf{d} S .
$$

Thus, the circulation around a fluid ring $c_{\tau}=\Psi_{\tau}(c)$ dragged along by the flow (where $\Psi_{\tau}$ is the family of diffeomorphisms generated by fluid four-velocity $\overrightarrow{\boldsymbol{u}}$ ) is not generally conserved:

$$
\begin{aligned}
\frac{d}{d \tau} \oint_{C_{\tau}} h \boldsymbol{u} & =\frac{d}{d \tau} \int_{S_{\tau}} \mathbf{d}(h \boldsymbol{u}) \\
& =\int_{S} £_{\overrightarrow{\boldsymbol{u}}} \mathbf{d}(h \boldsymbol{u})=\int_{S} \mathbf{d} T \wedge \mathbf{d} S .
\end{aligned}
$$

Hence, Kelvin's theorem has been commonly thought to not hold for baroclinic flows, except in a weaker form: the circulation computed initially along a fluid ring of constant temperature or specific entropy is conserved 32 , 104.

In lieu of a conserved circulation law, one may introduce the potential vorticity, defined in general relativity by selecting the scalar field in Eq. (2.34), or the negative Hamiltonian in Eq. (2.36), to coincide with specific 
entropy $S$ (i.e. setting $\boldsymbol{l}=\mathbf{d} S$ ), to obtain a flux conservation law of the form 3.49 :

$$
\nabla_{\alpha}\left(\nabla_{\beta} S \star \Omega^{\beta \alpha}\right)=0 .
$$

Using the continuity equation, this law can also be written in terms of a Lie derivative along fluid velocity and, as mentioned earlier, it is the relativistic generalization of Ertel's theorem obtained by Friedman [104] (see also Katz [105]).

Here, we take a different route, and show that Carter's framework [106] implies the existence of a strong circulation law. We have just shown above that an inviscid baroclinic fluid is a Hamiltonian system and, as such, must possess a Poincaré-Cartan integral invariant. Indeed, the exterior derivative of Eq. (3.43) implies that the canonical vorticity (3.19) is Lie-dragged by the canonical fluid velocity (2.7):

$$
£_{\overrightarrow{\boldsymbol{u}} / T} \mathbf{d}(h \boldsymbol{u})=0
$$

as dictated by Eq. (2.18). Hence, the circulation around a fluid ring $c_{\lambda}=\Psi_{\lambda}(c)$, obtained by moving each point of $c$ a canonical time $\lambda$ (cf. Eq. (3.40) ) along the flow through that point, is indeed conserved:

$$
\frac{d}{d \lambda} \oint_{c_{\lambda}} h \boldsymbol{u}=\frac{d}{d \lambda} \int_{S_{\lambda}} \mathbf{d}(h \boldsymbol{u})=\int_{S} £_{\overrightarrow{\boldsymbol{u}} / T} \mathbf{d}(h \boldsymbol{u})=0
$$

as dictated by Eqs. (2.20)-(2.21). Here, the circulation can be initially computed along an arbitrary fluid ring c. Thus, unlike the previous weak form, this circulation theorem is a strong form of Kelvin's theorem, applicable to baroclinic fluids.

It will be shown below that this circulation theorem can be further extended to barotropic or baroclinic, perfectly or poorly conducting, magnetofluids. These (new and old) circulation theorems are again special cases of the Poincaré-Cartan integral invariant (2.20). The fluid helicity, on the other hand, is not conserved for baroclinic fluids, as these systems are not uniformly canonical.

\section{Ideal magnetoflows}

\section{Maxwell equations}

Consider an electromagnetic field in $\mathscr{M}$, described by the electromagnetic 2-form $\boldsymbol{F}$, known as the Faraday tensor, satisfying the Maxwell equations which, in natural Heaviside-Lorentz units, read $\nabla_{\alpha}\left(\star F^{\alpha \beta}\right)=0$, $\nabla_{\alpha} F^{\alpha \beta}=J^{\beta}$ or

$$
\begin{gathered}
\mathbf{d} \boldsymbol{F}=0 \\
\mathbf{d} \star \boldsymbol{F}=\star \boldsymbol{J},
\end{gathered}
$$

where $\star \boldsymbol{F}$ is the 2-form Hodge-dual of $\boldsymbol{F}$, namely $\star F_{\alpha \beta}:=$ $\frac{1}{2} \epsilon_{\alpha \beta \gamma \delta} F^{\gamma \delta}$, and $\star \boldsymbol{J}$ is the 3 -form Hodge-dual of the 1form $\boldsymbol{J}$ associated with the electric 4-current $\overrightarrow{\boldsymbol{J}}$, namely $\star J_{\alpha \beta \gamma}:=\epsilon_{\alpha \beta \gamma \delta} J^{\delta}$.
The electric 4-current may be decomposed as $\overrightarrow{\boldsymbol{J}}=$ $e \overrightarrow{\boldsymbol{u}}+\overrightarrow{\boldsymbol{j}}$ where $e=-\boldsymbol{u} \cdot \overrightarrow{\boldsymbol{J}}$ is the proper charge density, $e \overrightarrow{\boldsymbol{u}}$ is the convection current and $\overrightarrow{\boldsymbol{j}}$ is the conduction current, satisfying $\boldsymbol{u} \cdot \vec{j}=0$. For an isotropically conducting medium, Ohm's law can be written as

$$
\boldsymbol{j}=\sigma \boldsymbol{E}
$$

where $\sigma$ is the conductivity of the medium and $\boldsymbol{E}$ is the electric field measured by an observer comoving with the fluid, given by Eq. (3.60) below. In the perfect conductivity limit, $\sigma \rightarrow \infty$, the electric field vanishes, $\boldsymbol{E} \rightarrow 0$. In the poor conductivity limit, $\sigma \rightarrow 0$, the conduction current vanishes, $\boldsymbol{j} \rightarrow 0$.

\section{Magnetohydrodynamic Euler equation}

The relativistic MHD-Euler equation can be obtained from the conservation law of energy-momentum,

$$
\nabla \cdot\left(\boldsymbol{T}^{\mathrm{fl}}+\boldsymbol{T}^{\mathrm{em}}\right)=0
$$

where $\boldsymbol{T}^{\mathrm{em}}$ is the energy-momentum tensor of the electromagnetic field:

$$
T_{\alpha \beta}^{\mathrm{em}}=F_{\gamma \alpha} F_{\beta}^{\gamma}-\frac{1}{4} F_{\gamma \delta} F^{\gamma \delta} g_{\alpha \beta} .
$$

This tensor is trace-free: $g^{\alpha \beta} T_{\alpha \beta}^{\mathrm{em}}=0$. Taking the divergence of Eq. 3.55) and using the Maxwell equations (3.52), one obtains the well known relation

$$
\nabla \cdot T^{\mathrm{em}}=-\boldsymbol{F} \cdot \overrightarrow{\boldsymbol{J}}
$$

Substituting Eqs. (3.11) and (3.56) into the conservation law (3.54) yields the MHD-Euler equation for baroclinic magnetofluids:

$$
\overrightarrow{\boldsymbol{u}} \cdot \mathbf{d}(h \boldsymbol{u})=T \mathbf{d} S+\frac{1}{\rho} \boldsymbol{F} \cdot \overrightarrow{\boldsymbol{J}} .
$$

As shown in Ref. [57], the specific form (3.57) is well adapted to the cases where the spacetime exhibits some symmetries. Projecting the MHD-Euler equation along $\overrightarrow{\boldsymbol{u}}$ yields $T £_{\overrightarrow{\boldsymbol{u}}} S=\frac{1}{\rho} \boldsymbol{E} \cdot \overrightarrow{\boldsymbol{J}}$. The right hand side of this equation, which represents Joule heating, vanishes in the limit of perfect conductivity, whence the flow is adiabatic:

$$
£_{\overrightarrow{\boldsymbol{u}}} S=\overrightarrow{\boldsymbol{u}} \cdot \nabla S=0 .
$$

For barotropic magnetofluids, the above equation simplifies to

$$
\overrightarrow{\boldsymbol{u}} \cdot \mathbf{d}(h \boldsymbol{u})=\frac{1}{\rho} \boldsymbol{F} \cdot \overrightarrow{\boldsymbol{J}}
$$

In the abscence of pressure and currents $(h \rightarrow 1$ and $\boldsymbol{J} \rightarrow 0$ ), this equation reduces to the geodesic equation, $\overrightarrow{\boldsymbol{u}} \cdot \mathbf{d} \boldsymbol{u}=0$, as expected. 


\section{Perfectly conducting magnetoflows}

The electric field 1-form $\boldsymbol{E}$ and the magnetic field vector $\vec{B}$ measured in the fluid rest-frame, by an observer of 4 -velocity $\overrightarrow{\boldsymbol{u}}$, are given in terms of $\boldsymbol{F}$ by

$$
\boldsymbol{E}=-\overrightarrow{\boldsymbol{u}} \cdot \boldsymbol{F}, \quad \boldsymbol{B}=\overrightarrow{\boldsymbol{u}} \cdot \star \boldsymbol{F}
$$

and satisfy

$$
\boldsymbol{E} \cdot \overrightarrow{\boldsymbol{u}}=0, \quad \boldsymbol{B} \cdot \overrightarrow{\boldsymbol{u}}=0 .
$$

Equivalently, we can decompose $\boldsymbol{F}$ into electric and magnetic parts with respect to the rest frame defined by the vector $\overrightarrow{\boldsymbol{u}}$, as

$$
\begin{aligned}
\boldsymbol{F} & =\boldsymbol{u} \wedge \boldsymbol{E}+\star(\boldsymbol{u} \wedge \boldsymbol{B}) \\
\star \boldsymbol{F} & =\star(\boldsymbol{u} \wedge \boldsymbol{E})-\boldsymbol{u} \wedge \boldsymbol{B} .
\end{aligned}
$$

The scalar invariants of the field are given by

$$
\begin{aligned}
\frac{1}{2} F^{\alpha \beta} F_{\alpha \beta} & =\overrightarrow{\boldsymbol{B}} \cdot \boldsymbol{B}-\overrightarrow{\boldsymbol{E}} \cdot \boldsymbol{E} \\
\frac{1}{2}\left(\star F^{\alpha \beta}\right) F_{\alpha \beta} & =\overrightarrow{\boldsymbol{B}} \cdot \boldsymbol{E} .
\end{aligned}
$$

In ideal MHD, one assumes that the fluid occupying the part $\mathcal{D} \subset \mathscr{M}$ of spacetime is a perfect conductor. By this, we mean that the observers comoving with the fluid measure a vanishing electric field. By virtue of Ohm's law (3.53), this expresses the infinite conductivity condition. From (3.60), this condition amounts to

$$
\boldsymbol{E}=\boldsymbol{F} \cdot \overrightarrow{\boldsymbol{u}}=0
$$

The electromagnetic field then reduces to

$$
\begin{aligned}
\boldsymbol{F} & =\star(\boldsymbol{u} \wedge \boldsymbol{B}) \\
\star \boldsymbol{F} & =-\boldsymbol{u} \wedge \boldsymbol{B}
\end{aligned}
$$

and the Maxwell equation (3.52a) simplifies to

$$
\mathbf{d} \star(\boldsymbol{u} \wedge \boldsymbol{B})=0 \Leftrightarrow \nabla_{\alpha}\left(u^{\alpha} B^{\beta}-u^{\beta} B^{\alpha}\right)=0 .
$$

This equation is a special case of Eq. (2.37), for reasons that will become clear below. In ideal MHD, one only has to evolve the magnetic field equation (3.67). The current has no dynamical degrees of freedom and is merely defined in terms of the magnetic field via Eq. (3.66a) and the Maxwell equation (3.52b). One then evolves the MHD-Euler equation (3.57) after evaluating the Lorentz force term in its right-hand side.

Alternatively, by writing $\boldsymbol{F}$ in terms of the electromagnetic potential 1-form $\boldsymbol{A}$,

$$
\boldsymbol{F}=\mathrm{d} \boldsymbol{A},
$$

one automatically satisfies the Maxwell equation (3.52a). The perfect conductivity condition (3.65) is then used to evolve the electromagnetic potential [107, 108]:

$$
\boldsymbol{u} \cdot \mathbf{d} \boldsymbol{A}=0 \Leftrightarrow u^{\alpha}\left(\nabla_{\alpha} A_{\beta}-\nabla_{\beta} A_{\alpha}\right)=0
$$

In $3+1$ dimensions, Eq. (3.67) is the curl of Eq. (3.69), as shown in $\S$ 凹D 11.

\section{Action of a magnetic field frozen into the flow}

A magnetic field frozen into the fluid, as defined by the perfect conductivity condition (3.65), is characterized by the action functional

$$
\mathcal{S}=\int_{\tau_{1}}^{\tau_{2}} A_{\alpha} \frac{d x^{\alpha}}{d \tau} d \tau
$$

where the electromagnetic potential $\boldsymbol{A}$ is considered a function of $x$ only. From the Lagrangian [27]

$$
L(x, u)=u^{\alpha} A_{\alpha}
$$

we finds that the canonical momentum 1-form (2.9b) is the electromagnetic potential

$$
p_{\alpha}=\frac{\partial L}{\partial u^{\alpha}}=A_{\alpha}
$$

and the canonical vorticity 2-form (2.17) is simply the Faraday tensor

$$
F=\mathbf{d} A
$$

Because the super-Hamiltonian (2.12) vanishes,

$$
H=0,
$$

the canonical equation of motion (2.14) takes the form of the perfect conductivity condition (3.69).

\section{Alfvén's theorem: conservation of magnetic flux}

If we express the Lie derivative of $\boldsymbol{F}$ along $\overrightarrow{\boldsymbol{u}}$ via the Cartan identity,

$$
£_{\vec{u}} \boldsymbol{F}=\overrightarrow{\boldsymbol{u}} \cdot \mathrm{d} \boldsymbol{F}+\mathrm{d}(\overrightarrow{\boldsymbol{u}} \cdot \boldsymbol{F}),
$$

and take into account the Maxwell equation (3.52a and the perfect conductivity condition (3.65), we get

$$
£_{\vec{u}} \boldsymbol{F}=0 .
$$

This result, which also follows from Eq. (2.18), is the geometrical expression of Alfvén's magnetic flux theorem: the magnetic flux through a fluid ring $c_{\tau}$ dragged along by the flow is conserved

$$
\frac{d}{d \tau} \oint_{c_{\tau}} \boldsymbol{A}=\frac{d}{d \tau} \int_{S_{\tau}} \boldsymbol{F}=\int_{S} £_{\overrightarrow{\boldsymbol{u}}} \boldsymbol{F}=0 .
$$

This follows directly from Eq. (2.21) for the Lagrangian (3.71) and is therefore simply a special case of the Poincaré-Cartan integral invariant (2.20). Intuitively, Alfvén's theorem is a consequence of perfect conductivity. If one attempts to change the magnetic field and thus the magnetic flux through the ring $c_{\tau}$ of fluid, then, in accordance with Lenz's law, induced currents will generate a compensatory magnetic field in an attempt to cancel the change of flux. In the limit of perfect conductivity, this cancellation is perfect and the flux is exactly conserved. 


\section{Magnetic helicity}

Since the super-Hamiltonian (3.74) is constant, the system is uniformly canonical, and the magnetic helicity,

$$
\boldsymbol{h}_{\mathrm{em}}:=\overrightarrow{\boldsymbol{A}} \cdot \star \boldsymbol{F},
$$

obtained by substituting Eqs. 3.72 and 3.73 into (2.38), is conserved

$$
\nabla_{\alpha} h_{\mathrm{em}}^{\alpha}=0
$$

by virtue of Eq. (2.39). This implies a corresponding global conservation of the integrated flux of $h_{\mathrm{em}}^{\alpha}$ across a spatial hypersurface, which amounts to the relativistic generalization of Woltjer's magnetic helicity [32, 70, 72].

\section{Einstein-Maxwell-Euler spacetimes}

The classical action describing an Einstein-MaxwellEuler spacetime $(\mathscr{M}, \boldsymbol{g})$, coupled with a perfect fluid carrying an electric current, is given by [60]

$$
\mathcal{A}=\int d^{4} x \sqrt{-g}\left(-\epsilon+\frac{1}{16 \pi} R-\frac{1}{4} F_{\alpha \beta} F^{\alpha \beta}+A_{\alpha} J^{\alpha}\right),
$$

where $R$ is the Ricci scalar. By writing $\boldsymbol{F}$ in terms of a 1-form potential $\boldsymbol{A}, \mathrm{Eq}$. (3.68), one satisfies the Maxwell equation (3.52a). Varying the action with respect to the metric $\boldsymbol{g}$ yields the Einstein equations; varying with respect to the electromagnetic 4-potential $\boldsymbol{A}$ yields the Maxwell equations (3.52b); and varying with respect to the fluid variables yields the MHD Euler equation (3.57).

Instead of imposing the perfect MHD condition after varying the action, one may incorporate it into the action. This can be done by replacing the action (3.80) with

$$
\begin{aligned}
\mathcal{A}=\int d^{4} x \sqrt{-g} & {\left[-\epsilon+\frac{1}{16 \pi} R-\frac{1}{2} B_{\alpha} B^{\alpha}\right.} \\
+ & \left.a_{\alpha} \nabla_{\beta}\left(B^{\alpha} u^{\beta}-B^{\beta} u^{\alpha}\right)\right]
\end{aligned}
$$

where the 1-form $\boldsymbol{a}$ is a Lagrange multiplier used to enforce the flux freezing condition (3.67). In writing the action functional above, we have taken into account Eq. (3.63) in order to evaluate the magnetic energy term. This action functional differs by a surface term from that of Bekenstein-Oron [29] which, in our notation, reads

$$
\mathcal{A}=\int d^{4} x \sqrt{-g}\left(-\epsilon+\frac{1}{16 \pi} R-\frac{1}{2} B_{\alpha} B^{\alpha}+b^{\alpha} F_{\alpha \beta} u^{\beta}\right) .
$$

Here, the Lagrange multiplier $\boldsymbol{b}$ is used to enforce the perfect conductivity condition (3.65) and is shown to be the curl of $\boldsymbol{a}$ as indicated by Eq. (3.85) below. Our action (3.81) closely resembles the non-relativistic action of Bekenstein-Oron [29], which is a more natural starting point and simplifies the discussion below. Variation of the action (3.81) with respect to the multiplier $\boldsymbol{a}$ yields the Maxwell equation (3.67), while variation with respect to the magnetic field $\boldsymbol{B}$ and integration by parts yields the equation

$$
\vec{u} \cdot \mathrm{d} a=-B
$$

The multiplier $\boldsymbol{a}$ may thus be thought of as an auxiliary field, with $\boldsymbol{B}$ the electric part of the 2 -form

$$
f=\mathrm{d} a
$$

[compare Eq. (3.82) with (3.60)]. Note that the above equation automatically satisfies the orthogonality condition (3.61). Comparing Eqs. (3.82) and (3.60), we infer that the Faraday tensor $\boldsymbol{F}$ must be related to the 2 -form $\boldsymbol{f}$ via a relation $\star \boldsymbol{F}=-\boldsymbol{f}+\boldsymbol{w}$ where $\boldsymbol{w}$ is some 2 -form satisfying $\overrightarrow{\boldsymbol{u}} \cdot \boldsymbol{w}=0$. Since $\boldsymbol{w}$ has no electric part, it can be written in terms of its magnetic part, $\boldsymbol{b}=\overrightarrow{\boldsymbol{u}} \cdot \star \boldsymbol{w}$, as $\boldsymbol{w}=\star(\boldsymbol{u} \wedge \boldsymbol{b})$. Taking Eq. 3.66b) into account, we infer that

$$
\boldsymbol{f}=\star(-\boldsymbol{F}+\boldsymbol{u} \wedge \boldsymbol{b})=\boldsymbol{u} \wedge \boldsymbol{B}+\star(\boldsymbol{u} \wedge \boldsymbol{b})
$$

That is, the 2-form 3.83 has an electric part given by Eq. (3.82) and a magnetic part given by the 1-form

$$
b=u \cdot \star \mathrm{d} a .
$$

As pointed out by Bekenstein and Oron [29], the theory has a $\mathrm{U}(1) \times \mathrm{U}(1)$ symmetry, since the observable field $\boldsymbol{B}$ remains invariant under gauge transformations $\boldsymbol{A} \rightarrow$ $\boldsymbol{A}+\mathbf{d} \Lambda$ and $\boldsymbol{a} \rightarrow \boldsymbol{a}+\mathbf{d} \lambda$.

Taking the exterior derivative of Eq. (3.84) yields the Maxwell equation (3.52b), with the Faraday tensor given by Eq. (3.66a and the current 'defined' by

$$
J^{\alpha}=\nabla_{\beta}\left(u^{\alpha} b^{\beta}-u^{\beta} b^{\alpha}\right)
$$

or

$$
\boldsymbol{J}=\star \mathbf{d} \star(\boldsymbol{u} \wedge \boldsymbol{b}) .
$$

This expression has been obtained in [29] via a lengthy route and will be referred to as the Bekenstein-Oron current. Note that the above expression automatically satisfies the continuity equation

$$
\nabla \cdot \vec{J}=-\star \mathbf{d} \star \boldsymbol{J}=0
$$

regardless of any assumption about $\boldsymbol{u}$ and $\boldsymbol{b}$. Physically, the above equation expresses the conservation of electric charge. The operator $\star \mathbf{d} \star$ is the codifferential and has been expressed as the divergence taken with the $\nabla$ connection. For convenience, let us introduce an auxiliary vector $\overrightarrow{\boldsymbol{q}}$ and an auxiliary 1 -form $\boldsymbol{\eta}$ defined by

$$
q^{\alpha}:=b^{\alpha} / \rho, \quad \eta_{\alpha}:=F_{\alpha \beta} q^{\beta}
$$

or

$$
\overrightarrow{\boldsymbol{q}}:=\overrightarrow{\boldsymbol{b}} / \rho, \quad \boldsymbol{\eta}:=\boldsymbol{F} \cdot \overrightarrow{\boldsymbol{q}}
$$


One may then use the continuity equation (3.12) to write the Bekenstein-Oron current (3.87) as

$$
\overrightarrow{\boldsymbol{J}}=£_{\overrightarrow{\boldsymbol{q}}}(\rho \overrightarrow{\boldsymbol{u}})+\rho \overrightarrow{\boldsymbol{u}}(\boldsymbol{\nabla} \cdot \overrightarrow{\boldsymbol{q}}) .
$$

This expression can be used to write the Lorentz force term in (3.104b) as

$$
\frac{1}{\rho} \boldsymbol{F} \cdot \overrightarrow{\boldsymbol{J}}=\frac{1}{\rho} \boldsymbol{F} \cdot £_{\overrightarrow{\boldsymbol{q}}}(\rho \overrightarrow{\boldsymbol{u}})=-\overrightarrow{\boldsymbol{u}} \cdot \mathbf{d} \boldsymbol{\eta} .
$$

The last equality follows from projecting the Cartan identity, $£_{\overrightarrow{\boldsymbol{q}}} \boldsymbol{F}=\overrightarrow{\boldsymbol{q}} \cdot \mathbf{d} \boldsymbol{F}+\mathbf{d}(\overrightarrow{\boldsymbol{q}} \cdot \boldsymbol{F})$, along the vector $\rho \overrightarrow{\boldsymbol{u}}$ and using Eq. (3.65). By virtue of the above equality, the MHD-Euler equation (3.57) takes the canonical form

$$
£_{\vec{u}}(h \boldsymbol{u}+\boldsymbol{\eta})+\mathbf{d} h=\overrightarrow{\boldsymbol{u}} \cdot \mathbf{d}(h \boldsymbol{u}+\boldsymbol{\eta})=T \mathbf{d} S
$$

which is valid for baroclinic magnetofluids. For barotropic magnetofluids, the above equation simplifies to

$$
£_{\overrightarrow{\boldsymbol{u}}}(h \boldsymbol{u}+\boldsymbol{\eta})+\mathbf{d} h=\overrightarrow{\boldsymbol{u}} \cdot \mathbf{d}(h \boldsymbol{u}+\boldsymbol{\eta})=0 .
$$

The last equality was obtained by Bekenstein et al. 29, 31 .

The tensor or vector calculus-based derivations in Ref. [29, 31] did not clarify the generality of this approach. In particular, one may question whether the Bekenstein-Oron ansatz (3.87) for the current is generic enough to accommodate any given ideal MHD flow. This question boils down to whether Eq. (3.82) can be solved for any given magnetofluid configuration with magnetic field $\boldsymbol{B}$ and 4-velocity $\overrightarrow{\boldsymbol{u}}$. The answer may be obtained by using the Cartan identity to write Eq. (3.82) as $£_{\overrightarrow{\boldsymbol{u}}} \boldsymbol{a}-\mathbf{d}(\overrightarrow{\boldsymbol{u}} \cdot \boldsymbol{a})=-\boldsymbol{B}$ and using the gauge freedom in $\boldsymbol{a}$ to set $\overrightarrow{\boldsymbol{u}} \cdot \boldsymbol{a}=0$ (this gauge condition can be shown to be preserved by the flow if satisfied initially). The resulting differential equation, $£_{\overrightarrow{\boldsymbol{u}}} \boldsymbol{a}=-\boldsymbol{B}$, is always solvable along the integral curves of $\overrightarrow{\boldsymbol{u}}$. We have thus shown that no loss of generality is entailed in the Bekenstein-Oron description of ideal MHD flows. For perfectly conducting magnetofluids, the Einstein-Maxwell-Euler action (3.80) may always be replaced by the action 3.81, and the MHD-Euler equation (3.57) may always be replaced by Eq. 3.92).

\section{Hamilton's principle for a barotropic magnetofluid element}

Carter [27] has allowed the possibility that the perfect fluid be charged. His approach is valid for poorly conducting fluids, but has been considered inapplicable to conducting magnetofluids [30]. Nevertheless, it is shown below that Carter's framework can in fact accommodate perfectly conducting fluids in the context of BekensteinOron magnetohydrodynamics. For a barotropic, perfectly conducting magnetofluid, we generalize the action (3.16) as follows:

$$
\mathcal{S}=\int_{\tau_{1}}^{\tau_{2}}\left(-h \sqrt{-g_{\alpha \beta} \frac{d x^{\alpha}}{d \tau} \frac{d x^{\beta}}{d \tau}}+\eta_{\alpha} \frac{d x^{\alpha}}{d \tau}\right) d \tau
$$

with Lagrangian

$$
L(x, u)=-h \sqrt{-g_{\alpha \beta} u^{\alpha} u^{\beta}}+\eta_{\alpha} u^{\alpha}
$$

and with $\boldsymbol{\eta}$ given by Eq. 3.89). The canonical velocity and momentum of a magnetofluid element are given by

$$
\begin{aligned}
& u^{\alpha}=\frac{d x^{\alpha}}{d \tau} \\
& p_{\alpha}=\frac{\partial L}{\partial u^{\alpha}}=h u_{\alpha}+\eta_{\alpha} .
\end{aligned}
$$

Alternatively, one may introduce a Lagrangian which generalizes that of Carter, Eq. (3.20):

$$
L(x, u)=\frac{1}{2} h g_{\alpha \beta} u^{\alpha} u^{\beta}-\frac{1}{2} h+\eta_{\alpha} u^{\alpha} .
$$

The associated Hamiltonian,

$$
H(x, \pi)=\frac{1}{2 h} g^{\alpha \beta}\left(p_{\alpha}-\eta_{\alpha}\right)\left(p_{\beta}-\eta_{\beta}\right)+\frac{1}{2} h,
$$

vanishes on-shell, so the Hamilton equation (2.14) yields the MHD-Euler equation in the Bekenstein-Oron form, Eq. (3.93).

\section{Conservation of circulation in barotropic magnetoflows}

The canonical momentum 1-form of a barotropic ideal magnetofluid-element is given by Eq. 3.96b. Then, the Poincaré 2-form (2.17) amounts to the canonical vorticity 2-form:

$$
\boldsymbol{\Omega}=\mathbf{d}(h \boldsymbol{u}+\boldsymbol{\eta}) .
$$

Then, the Cartan identity, combined with Eq. 3.92 and the identity $\mathbf{d}^{2}=0$, yields

$$
£_{\overrightarrow{\boldsymbol{u}}} \Omega=0 .
$$

This equation implies that the canonical vorticity of a barotropic, perfectly conducting magnetofluid is preserved by the flow. This leads to a generalization of Kelvin's theorem to magnetized fluids.

Indeed, for the system (3.94), the Poincaré-Cartan theorem (2.21) implies that the circulation through a ring $c_{\tau}$ dragged along by the flow is conserved:

$$
\frac{d}{d \tau} \oint_{c_{\tau}}(h \boldsymbol{u}+\boldsymbol{\eta})=0 .
$$

This law follows directly from Eq. (3.100 and was first obtained by Bekenstein and Oron [29, 30]. It is a generalization of the relativistic Kelvin circulation theorem (3.48) (which is recovered in the non-magnetic limit $\boldsymbol{\eta}=0$ ) to ideal MHD. The most interesting feature of this conservation law is that it is exact in time-dependent spacetimes, with gravitational and electromagnetic waves carrying energy and angular momentum away from a system. In particular, oscillating stars and radiating binaries, if modeled as barotropic magnetofluids with no viscosity, resistivity or other dissipation, exactly conserve circulation. 


\section{Ideal magnetofluid helicity}

Since the super-Hamiltonian (3.98) is constant (zero), the system is uniformly canonical, and helicity is conserved: Substituting Eq. 3.96b into Eq. (2.38) yields the magnetolfuid helicity

$$
\boldsymbol{h}_{\mathrm{mfl}}:=(h \overrightarrow{\boldsymbol{u}}+\boldsymbol{\eta}) \cdot \star \boldsymbol{\Omega}
$$

which, by virtue of Eq. 2.39), is conserved:

$$
\nabla_{\alpha}\left[\left(h u_{\beta}+\eta_{\alpha}\right) \star \Omega^{\beta \alpha}\right]=0 .
$$

This implies a corresponding global conservation of the integrated flux of $h_{\mathrm{mfl}}^{\alpha}$ across a spatial hypersurface. One may proceed analogously to Eq. (3.26) to obtain a conserved volume integral, which amounts to the generalization of Moffat's fluid helicity [32, 71, 72] to ideal GRMHD.

\section{A canonical evolution scheme for ideal MHD}

In binary neutron-star inspiral, the temperature is much lower than the Fermi temperature, and heat conduction, viscosity and resistivity can be neglected [60]. The fluid may then be approximated as barotropic, adiabatic, inviscid and perfectly conducting. In general relativity, such fluids are described by the ideal MHD equations (3.69) and (3.59):

$$
\begin{aligned}
u^{\alpha}\left(\nabla_{\alpha} A_{\beta}-\nabla_{\beta} A_{\alpha}\right) & =0 \\
u^{\alpha}\left[\nabla_{\alpha}\left(h u_{\beta}\right)-\nabla_{\beta}\left(h u_{\alpha}\right)\right] & =\frac{1}{\rho} F_{\beta \alpha} J^{\alpha} .
\end{aligned}
$$

coupled to the continuity equation (3.12). One can evolve Eq. (3.69) for the electromagnetic potential and compute the Faraday tensor via Eq. (3.68). In ideal MHD, as mentioned earlier, the current lacks dynamical degrees of freedom and is merely 'defined' in terms of the electromagnetic potential via the Maxwell equation (3.52b). One then evolves the MHD-Euler equation (3.104b) after evaluating the Lorentz force term in its right-hand side.

In a chart $\left\{t, x^{i}\right\}$, the above system can be written in $3+1$ hyperbolic form 4 as

$$
\begin{aligned}
\partial_{t} A_{i}-\partial_{i} A_{t}+v^{j}\left(\partial_{j} A_{i}-\partial_{i} A_{j}\right) & =0 \\
\partial_{t} \pi_{i}-\partial_{i} \pi_{t}+v^{j}\left(\partial_{j} \pi_{i}-\partial_{i} \pi_{j}\right) & =f_{i}
\end{aligned}
$$

where $v^{i}=u^{i} / u^{t}=d x^{i} / d t$ is the fluid velocity measured in local coordinates, $\pi_{\alpha}=h u_{\alpha}$ denotes a (non-canonical) momentum 1-form and $f_{\alpha}=\left(\rho u^{t}\right)^{-1} F_{\alpha \beta} J^{\beta}$ denotes the Lorentz force per particle. The curl of the evolution equation (3.105a) is an evolution equation for the magnetic

\footnotetext{
4 The four-momenta $\left\{A_{t}, A_{i}\right\}$ and $\left\{\pi_{t}, \pi_{i}\right\}$ can be expressed in terms of quantities normal or tangent to a $t=$ constant hypersurface in a manner described in Ref. [66.
}

field. In particular, the exterior derivatives of the system (3.105) yield an evolution system for the spatial parts of the 2-forms $\boldsymbol{F}=\mathbf{d} \boldsymbol{A}$ and $\boldsymbol{W}=\mathbf{d} \boldsymbol{\pi}$. In flux-conservative form, this system reads:

$$
\begin{aligned}
\partial_{t} F_{j k}+\partial_{i}\left(\delta_{j k}^{i l} v^{m} F_{m l}\right) & =0 \\
\partial_{t} W_{j k}+\partial_{i}\left[\delta_{j k}{ }^{i l}\left(v^{m} W_{m l}-f_{l}\right)\right] & =0
\end{aligned}
$$

where $\delta_{j k}^{i l}=\epsilon_{j k n} \epsilon^{i l n}=\delta_{j}^{i} \delta_{k}^{l}-\delta_{k}^{i} \delta_{j}^{l}$ is the generalized Kronecker delta. Eq. (3.106a) is an evolution equation, equivalent5 to Eq. (3.67), for the magnetic field. Numerical evolution of the latter typically requires techniques such as hyperbolic divergence cleaning or constrained transport to avoid error accumulation from a finite magnetic divergence 109]. Such numerical schemes can also be applied to evolving the system (3.106) [as well as the system (3.110) below]. Etienne et al. [107, 108] have performed GRMHD simulations that directly evolve the electromagnetic potential $\boldsymbol{A}$ by means of Eq. 3.105a [or Eq. (3.109a $]$. The magnetic field is then computed from the curl of the vector potential and has zero divergence by construction. This numerical scheme can also be applied to evolving the system (3.109) below, which is based on the Bekenstein-Oron formulation.

Eqs. 3.104 - 3.106 constitute the usual formulation of ideal MHD for barotropic magnetofluids. As shown earlier, the Bekenstein-Oron description of ideal MHD allows one to replace the MHD-Euler equation (3.59) by the system of equations (3.82) and (3.93), namely

$$
\begin{aligned}
u^{\alpha}\left(\nabla_{\alpha} A_{\beta}-\nabla_{\beta} A_{\alpha}\right) & =0 \\
u^{\alpha}\left(\nabla_{\alpha} a_{\beta}-\nabla_{\beta} a_{\alpha}\right) & =-B_{\beta} \\
u^{\alpha}\left(\nabla_{\alpha} p_{\beta}-\nabla_{\beta} p_{\alpha}\right) & =0
\end{aligned}
$$

where

$$
\boldsymbol{p}=h \boldsymbol{u}+\boldsymbol{\eta}
$$

is the canonical momentum 1-form of a magnetofluid element, as shown in the next section.

In a chart $\left\{t, x^{i}\right\}$, the above system can be written in $3+1$ canonical hyperbolic form as

$$
\begin{aligned}
\partial_{t} A_{i}-\partial_{i} A_{t}+v^{j}\left(\partial_{j} A_{i}-\partial_{i} A_{j}\right) & =0 \\
\partial_{t} a_{i}-\partial_{i} a_{t}+v^{j}\left(\partial_{j} a_{i}-\partial_{i} a_{j}\right) & =-B_{i} \\
\partial_{t} p_{i}-\partial_{i} p_{t}+v^{j}\left(\partial_{j} p_{i}-\partial_{i} p_{j}\right) & =0
\end{aligned}
$$

This system may be evolved analogously to the system (3.104). One evolves the first equation for $\boldsymbol{A}$ and computes the magnetic field $\boldsymbol{B}=\overrightarrow{\boldsymbol{u}} \cdot \star \mathbf{d} \boldsymbol{A}$. With this source, one evolves the second equation for $\boldsymbol{a}$ and computes the auxiliary field $\boldsymbol{b}=\overrightarrow{\boldsymbol{u}} \cdot \star \mathbf{d} \boldsymbol{a}$. Finally, one solves the last equation of the above system, taking Eqs. (3.89) and (3.108) into account, to evolve the hydromagnetic flow.

\footnotetext{
5 Unlike Eq. 3.67) which contains the metric and its connection, Eq. 3.106a contains no such dependence, yet both equations are equivalent and exact in curved spacetime.
} 
The spatial exterior derivatives of the system (3.109) yield an evolution system for the spatial parts of the 2forms (3.73), 3.83) and 3.99). In flux-conservative form, this system reads:

$$
\begin{aligned}
& \partial_{t} F_{j k}+\partial_{i}\left(\delta_{j k}^{i l} v^{m} F_{m l}\right)=0 \\
& \partial_{t} f_{j k}+\partial_{i}\left[\delta_{j k}^{i l}\left(v^{m} f_{m l}+B_{l}\right)\right]=0 \\
& \partial_{t} \Omega_{j k}+\partial_{i}\left(\delta_{j k}^{i l} v^{m} \Omega_{m l}\right)=0
\end{aligned}
$$

As mentioned above, the numerical schemes developed for the systems (3.105) or 3.106) can also be applied to evolving the systems 3.109 or 3.110. Note that these systems were obtained from equations involving only exterior derivatives, and thus do not involve the spacetime metric or its connection. Thus, these systems are independent of gravity theory and they can be shown to be valid as writter 6 even in the Newtonian limit. This is generally true for equations of motion written in Euler-Lagrange or in Hamiltonian form, cf. 66 for details. For nonmagnetic fluids, Eq. (3.109c) was obtained from a $3+1$ constrained Hamiltonian formulation of the Euler equation in Ref. [66], where it was shown to be strongly hyperbolic. Other strongly hyperbolic formulations of the relativistic Euler equation include the Valencia formulation [11] and the symmetric hyperbolic Fraudendiner-Walton formulation [110 113]. The hyperbolicity of the evolution system 3.109 is the subject of future work. A notable feature of the canonical evolution system 3.107 is that it manifestly preserves magnetic flux and circulation, owing to its symplecic structure. Eq. 3.109a can also be obtained from a constrained Hamiltonian. Symplectic evolution schemes based on the Hamiltonians of Eqs. (3.109a and (3.109c) are expected to numerically preserve such properties. Moreover, if the system admits a Noether symmetry, this canonical form quickly gives rise to first integrals as discussed below.

\section{Magnetars with helical symmetry}

As an example, let us consider a helically symmetric rigidly rotating system, such as a rigidly rotating magnetar triaxially deformed by its off-axis frozen magnetic field. The flow field may then be written in the form of Eq. (3.31). Let us assume that all observable fields (such as $h, \boldsymbol{u}, \boldsymbol{B}, \boldsymbol{F}, \boldsymbol{g})$ are helically symmetric, that is, their Lie derivatives along the helical Killing vector $\overrightarrow{\boldsymbol{k}}$, given by Eq. (3.32), vanish.

Using gauge freedom, one can always find a gauge class for which the electromagnetic potential $\boldsymbol{A}$ inherits the Killing symmetries of $\boldsymbol{F}=\mathbf{d} \boldsymbol{A}$ [57, 114 116]. Then, using

\footnotetext{
${ }^{6}$ With $-p_{t}$ replaced by the constrained Hamiltonian $H\left(p_{i}, x^{j}\right)$, and similarly for $-A_{t}$ and $-a_{t}$, Eqs. 3.109 are formally valid in both $3+1$ general relativity and in Newtonian gravity [66].
}

Eq. (3.31) and the Cartan identity, $£_{\vec{k}} \boldsymbol{A}=\overrightarrow{\boldsymbol{k}} \cdot \mathbf{d} \boldsymbol{A}+\mathbf{d}(\overrightarrow{\boldsymbol{k}}$. $\boldsymbol{A})=0$, we find that Eq. 3.107a has the first integral

$$
\boldsymbol{A} \cdot \overrightarrow{\boldsymbol{k}}=A_{t}+\Omega A_{\varphi}=\text { constant }
$$

Similarly, using $£_{\overrightarrow{\boldsymbol{k}}} \boldsymbol{a}=\overrightarrow{\boldsymbol{k}} \cdot \mathbf{d} \boldsymbol{a}+\mathbf{d}(\overrightarrow{\boldsymbol{k}} \cdot \boldsymbol{a})$ and imposing the gauge condition $\overrightarrow{\boldsymbol{k}} \cdot \boldsymbol{a}=0$ allows one to write Eq. 3.107b as $£_{\overrightarrow{\boldsymbol{k}}} \boldsymbol{a}=-\boldsymbol{B} / u^{t}$. This equation has the simple solution

$$
\boldsymbol{a}=-\boldsymbol{B} t / u^{t},
$$

where the scalar field $t$ satisfies $t^{\alpha} \nabla_{\alpha} t=1$, so that $£_{\vec{k}} t=$ $\left(\partial_{t}+\Omega \partial_{\varphi}\right) t=1$. Note that the auxiliary fields $\boldsymbol{a}$ and $\boldsymbol{b}=\overrightarrow{\boldsymbol{u}} \cdot \star \mathbf{d} \boldsymbol{a}$ are not observable and need not satisfy helical symmetry (cf. Appendix B). Finally, Eq. (3.31) and the Cartan identity allow one to write Eq. (3.107c) in the form of Eq. (3.34), which has the first integral

$$
\boldsymbol{p} \cdot \overrightarrow{\boldsymbol{k}}+f=-h / u^{t}+f=\text { constant. }
$$

The first integrals (3.111) and 3.113 are consequences of stationarity in an inertial $(\Omega=0)$ or rotating $(\Omega>0)$ frame and, like Eq. 3.35, can be considered generalizations of von Zeipel's law to relativistic magnetoflows. The scalar $f$ is such that $\mathbf{d} f=-£_{\vec{k}} \boldsymbol{p}$ or, by virtue of Eq. (3.108),

$$
\mathbf{d} f=-£_{\vec{k}} \boldsymbol{\eta} .
$$

The right-hand side of this equation is proportional to the Lorentz force. One way to see this is to act with $£_{\boldsymbol{k}}$ on Eq. 3.89 ,

$$
\boldsymbol{\eta}=\mathbf{d} \boldsymbol{A} \cdot \overrightarrow{\boldsymbol{b}} / \rho=\left(B^{2} / \rho\right) \boldsymbol{u}-\mathbf{d} \boldsymbol{a} \cdot \overrightarrow{\boldsymbol{B}} / \rho,
$$

and use Eq. (3.112), yielding

$$
£_{\overrightarrow{\boldsymbol{k}}} \boldsymbol{\eta}=\mathbf{d}\left(\boldsymbol{B} / u^{t}\right) \cdot \overrightarrow{\boldsymbol{B}} / \rho .
$$

Eq. (3.114 then implies that the Lorentz force must be the gradient of a scalar potential $f$ in order for helically symmetric corotating configurations solutions to exist. This equation is subject to the integrability condition

$$
\mathbf{d} £_{\vec{k}} \boldsymbol{\eta}=-\mathbf{d}^{2} f=0 .
$$

which constitutes a restriction on the magnetic field $\boldsymbol{B}$ on which $\boldsymbol{\eta}$ depends. By virtue of Eq. (3.116), the above condition becomes

$$
\mathbf{d}\left(\boldsymbol{B} / u^{t}\right) \wedge \mathbf{d}(\boldsymbol{B} / \rho)=0 .
$$

For corotating helically symmetric magnetoflows, the system of nonlinear partial differential equations (3.107)

\footnotetext{
${ }^{7}$ Note that, on-shell, the fields $\boldsymbol{\eta}, \boldsymbol{A}$ and $\overrightarrow{\boldsymbol{b}}$ are independent of $\overrightarrow{\boldsymbol{u}}$, whereas $\overrightarrow{\boldsymbol{B}}$ depends on $\overrightarrow{\boldsymbol{u}}$ via Eq. (3.60).
} 
has been reduced to the system of algebraic equations (3.111)-(3.113) and the partial differential equation (3.114). The Newtonian analogue of Eq. (3.117) has been considered in Ref. 117]. A full description of a triaxial magnetar requires specifying boundary (or junction) conditions at the stellar surface for the electromagnetic field, as well as an induced surface current (associated with the fact that the condition (3.111) applies inside the star but not in the vacuum outside the surface). This is beyond the scope of this paper and a subject of future work.

\section{Hamilton's principle for a baroclinic magnetofluid element}

For a baroclinic, perfectly conducting magnetofluid, we consider the action

$$
\mathcal{S}=\int_{\lambda_{1}}^{\lambda_{2}}\left(-h \sqrt{-g_{\alpha \beta} \frac{d x^{\alpha}}{d \lambda} \frac{d x^{\beta}}{d \lambda}}+\eta_{\alpha} \frac{d x^{\alpha}}{d \lambda}+S\right) d \lambda
$$

with $\boldsymbol{\eta}$ given by Eq. (3.89). Like its non-magnetic limit (3.39), the above functional is parametrized in terms of canonical time $\lambda$, cf. Eq. (3.40). The Lagrangian of a magnetofluid element

$$
L(x, v)=-h \sqrt{-g_{\alpha \beta} v^{\alpha} v^{\beta}}+\eta_{\alpha} v^{\alpha}+S
$$

is associated with a canonical velocity and canonical momentum

$$
\begin{aligned}
& v^{\alpha}=\frac{d x^{\alpha}}{d \lambda}=\frac{1}{T} \frac{d x^{\alpha}}{d \tau}=\frac{1}{T} u^{\alpha} \\
& p_{\alpha}=\frac{\partial L}{\partial v^{\alpha}}=T h v_{\alpha}+\eta_{\alpha}=h u_{\alpha}+\eta_{\alpha} .
\end{aligned}
$$

On-shell, by virtue of Eqs. (3.14), (3.65) and (3.89), the Lagrangian takes the value $L=-g / T=-h / T+S$ and, by virtue of Eq. (2.12), the super-Hamiltonian takes the value $H=-S$. The Euler-Lagrange equation (2.10) thus becomes

$$
£_{\overrightarrow{\boldsymbol{u}} / T}(h \boldsymbol{u}+\boldsymbol{\eta})=\mathbf{d}(S-h / T)
$$

and the Hamilton equation (2.14) becomes

$$
\frac{\vec{u}}{T} \cdot \mathbf{d}(h \boldsymbol{u}+\boldsymbol{\eta})=\mathbf{d} S
$$

These equations are related via the Cartan identity and are equivalent expressions of the MHD-Euler equation (3.92).

Alternatively, one may generalize Carter's Lagrangian (3.45) to perfectly conducting baroclinic magnetofluids: the resulting Lagrangian

$$
L(x, v)=\frac{1}{2} T h g_{\alpha \beta} v^{\alpha} v^{\beta}+\eta_{\alpha} v^{\alpha}-\frac{1}{2}\left(\frac{g}{T}-S\right)
$$

is associated with the same canonical velocity and momentum (3.96) and leads also to the equation of motion
(3.122). The Legendre transformation (2.12) yields the super-Hamiltonian

$$
H(x, p)=\frac{1}{2 T h} g^{\alpha \beta}\left(p_{\alpha}-\eta_{\alpha}\right)\left(p_{\beta}-\eta_{\beta}\right)+\frac{h}{2 T}-S,
$$

which leads to the canonical equation of motion (3.123). Note that the 1-form $\boldsymbol{\eta}$, defined by Eq. (3.89) or (3.115), is consider independent of the four-velocity $\overrightarrow{\boldsymbol{u}}$.

\section{Conservation of circulation in baroclinic magnetoflows}

Like their nonmagnetic counterparts, baroclinic magnetoflows do not Lie-drag the vorticity (3.99): the exterior derivative of Eq. (3.92) reads

$$
£_{\overrightarrow{\boldsymbol{u}}} \mathbf{d}(h \boldsymbol{u}+\boldsymbol{\eta})=\mathbf{d} T \wedge \mathbf{d} S .
$$

Thus, as in Eq. (3.48), the circulation around a magnetofluid ring dragged along by the flow is not generally conserved, except in a weak sense, i.e. for rings of constant specific entropy or temperature.

Nevertheless, like their nonmagnetic counterparts, ideal baroclinic magnetoflows are Lie-dragged by the canonical fluid velocity (3.96a):

$$
£_{\vec{u} / T} \mathbf{d}(h \boldsymbol{u}+\boldsymbol{\eta})=0
$$

as dictated by Eq. (3.122), and this leades to a strong conservation law. In particular, the circulation around a magnetofluid ring $c_{\lambda}=\Psi_{\lambda}(c)$, obtained by moving each point of $c$ a thermal time $\lambda$ (cf. Eq. (3.40) ) along the flow through that point, is indeed conserved:

$$
\begin{aligned}
\frac{d}{d \lambda} \oint_{c_{\lambda}} h \boldsymbol{u}+\boldsymbol{\eta} & =\frac{d}{d \lambda} \int_{S_{\lambda}} \mathbf{d}(h \boldsymbol{u}+\boldsymbol{\eta}) \\
& =\int_{S} £_{\overrightarrow{\boldsymbol{u}} / T} \mathbf{d}(h \boldsymbol{u}+\boldsymbol{\eta})=0 .
\end{aligned}
$$

Here, the circulation can be initially computed along an arbitrary fluid ring $c$. This conservation of circulation law generalizes the Bekenstein-Oron law (3.101) to baroclinic magnetofluids. The conserved circulation is the Poincaré-Cartan integral invariant of the Hamiltonian system described by the action (3.119). Although it has not appeared in the literature before, it is a special case of Eqs. (2.20) and (2.21), like all circulation integrals presented earlier.

A very similar conservation of circulation law can be obtained for a poorly conducting fluid, simply by replacing $\eta_{\alpha}$ with $e A_{\alpha}$, where $e$ is the net charge per fluid element, in the action (3.119) and all equations that follow from it (cf. 27] for poorly conducting barotropic fluids). Although conservation of circulation holds in the limits of infinite or zero conductivity, we have not been able to obtain such a law for finite conductivity. This may be attributed to the fact that, for finite conductivity, the MHD-Euler equation (3.57) does not follow from a Hamiltonian and, equivalently, does not possess a Poincaré-Cartan integral invariant. 


\section{E. The geometry of barotropic flows}

\section{Hydrodynamic flows as geodesics in a Riemann space}

In Riemann geometry, the line element is given by the quadratic expression

$$
d \mathcal{S}^{2}=-\gamma_{\alpha \beta}(x) d x^{\alpha} d x^{\beta}
$$

where $\gamma_{\alpha \beta}(x)$ is a Lorentzian metric on a Riemannian manifold $\mathcal{M}$. The distance between two points (or events) 1 and 2 is then given by the integral

$$
\mathcal{S}=-\int_{1}^{2} \sqrt{-\gamma_{\alpha \beta}(x) d x^{\alpha} d x^{\beta}}=-\int_{\tau_{1}}^{\tau_{2}} \sqrt{-\gamma_{\alpha \beta}(x) \dot{x}^{\alpha} \dot{x}^{\beta}} d \tau
$$

where $\dot{x}^{\alpha}=d x^{\alpha} / d \tau$ is the velocity. This functional is independent of the parameter $\tau$.

It was demonstrated above that if a perfect fluid is barotropic, then the motion of a fluid element is conformally geodesic. In particular, Synge [17] and Lichnerowicz [18] have shown that the motions of fluid elements in a barotropic fluid are geodesics of a manifold $\mathcal{M}$ with metric

$$
\gamma_{\alpha \beta}(x)=h(x)^{2} g_{\alpha \beta}(x)
$$

conformally related to the spacetime metric $g_{\alpha \beta}(x)$. As shown earlier, such fluid motions can indeed be obtained from the action (3.16), which represents the arc length (3.130) between two events, and is independent of the parameter $\tau$.

\section{Magnetohydrodynamic flows as geodesics in a Finsler space}

One may think that the above result of Synge and Lichnerowicz ceases to apply in MHD, due to the highly complicated nature of the MHD-Euler equation (3.57). Surprisingly, however, the above results can be extended to magnetofluids that are barotropic and perfectly conducting. Such flows are described by the action (3.94), which is independent of the parameter $\tau$, and are geodesic in a Finsler (rather than Riemann) space [118-120]. In particular, in the context of Finsler spaces, Eq. (3.94) has similarities with the Randers metric [121, 122].

As pointed out by Chern [118], Finsler geometry is simply Riemann geometry without the quadratic restriction (3.129). In Finsler geometry, the line-element is replaced by the general expression

$$
d \mathcal{S}=L(x, d x) .
$$

where $L: \mathbb{R}^{2} \rightarrow \mathbb{R}$ is an arbitrary function that can be identified with the Lagrangian. Then, the distance between two points is given by

$$
\mathcal{S}=\int_{1}^{2} L(x, d x)=\int_{\tau_{1}}^{\tau_{2}} L(x, \dot{x}) d \tau
$$

where the last equality holds iff the function $L(x, \dot{x})$ is homogeneous of degree 1 in the velocity $\dot{x}^{\alpha}=d x^{\alpha} / d \tau$ :

$$
L(x, \kappa \dot{x})=\kappa L(x, \dot{x}) \quad \forall \kappa>0 .
$$

Lagrangians with this homogeneity property give rise to a parametrization-independent action functional, and lay at the foundation of Finsler geometry.

The Lagrangian in the perfect magnetofluid action functional 3.94 satisfies the above homogeneity property and can thus be expressed in the form of arc length in a Finsler space. To show this explicitly, we proceed as follows. Following Chern [118], we consider the projectivized tangent bundle $\mathcal{P} \mathcal{T} \mathcal{M}$ (i.e. the bundle of line elements) of the manifold $\mathcal{M}$. All geometric quantities constructed from the Lagrangian $L$ are homogeneous of degree zero in $\dot{x}^{\alpha}$ and thus naturally live on $\mathcal{P} \mathcal{T} \mathcal{M}$, although $L$ itself does not. Let $\left\{x^{\mu}\right\}$ be local coordinates on $\mathcal{M}$. Express tangent vectors as $\dot{x}^{\mu} \partial_{\mu}$ so that $\left\{x^{\mu}, \dot{x}^{\mu}\right\}$ can be used as local coordinates of $\mathcal{T} \mathcal{M}$ and, with $\dot{x}^{\mu}$ homogeneous, as local coordinates on $\mathcal{P} \mathcal{T} \mathcal{M}$. Euler's theorem of homogeneous functions (c.f. Appendix $\mathrm{A}$ ) can be used to show that

$$
L(x, \dot{x})=\underbrace{\frac{\partial L}{\partial \dot{x}^{\alpha}}}_{p_{\alpha}} \dot{x}^{\alpha}=-(\underbrace{\frac{1}{2} \frac{\partial^{2} L^{2}}{\partial \dot{x}^{\alpha} \partial \dot{x}^{\beta}}}_{-\gamma_{\alpha \beta}} \dot{x}^{\alpha} \dot{x}^{\beta})^{1 / 2} .
$$

The Hessian

$$
\gamma_{\alpha \beta}(x, \dot{x}):=-\frac{1}{2} \frac{\partial^{2} L^{2}}{\partial \dot{x}^{\alpha} \partial \dot{x}^{\beta}}
$$

plays the role of a metric on $\mathcal{P} \mathcal{T} \mathcal{M}$. This is a metric in a Finsler (rather than Riemann) space, as it depends on velocity in addition to position. A Finslerian metric is homogeneous of degree zero in the velocity:

$$
\gamma_{\alpha \beta}(x, \kappa \dot{x})=\gamma_{\alpha \beta}(x, \dot{x}) \quad \forall \kappa>0,
$$

as implied by Eqs. (3.134) and (3.136). That is, the Finslerian metric $\gamma_{\alpha \beta}(x, \dot{x})$ depends on the direction, but not magnitude, of the velocity $\dot{x}^{\alpha}$. The line element (3.132) can then be written as

$$
d \mathcal{S}^{2}=-\gamma_{\alpha \beta}(x, \dot{x}) d x^{\alpha} d x^{\beta}
$$

and the functional 3.133 becomes

$$
\begin{aligned}
\mathcal{S} & =-\int_{1}^{2} \sqrt{-\gamma_{\alpha \beta}(x, \dot{x}) d x^{\alpha} d x^{\beta}} \\
& =-\int_{\tau_{1}}^{\tau_{2}} \sqrt{-\gamma_{\alpha \beta}(x, \dot{x}) \dot{x}^{\alpha} \dot{x}^{\beta}} d \tau
\end{aligned}
$$

For our particular application, substituting the ideal MHD Lagrangian (3.95) into the definition (3.136) yields

$$
\begin{aligned}
\gamma_{\alpha \beta}(x, \dot{x})= & h^{2} g_{\alpha \beta}-\eta_{\alpha} \eta_{\beta}-h\left(\eta_{\alpha} u_{\beta}+\eta_{\beta} u_{\alpha}\right) \\
& -h q_{\alpha \beta} \eta_{\gamma} u^{\gamma},
\end{aligned}
$$


where $u^{\alpha}=\dot{x}^{\alpha}\left(-g_{\beta \gamma} \dot{x}^{\beta} \dot{x}^{\gamma}\right)^{-1 / 2}$ is the unit vector along $\dot{x}^{\alpha}, q_{\alpha \beta}=g_{\alpha \beta}+u_{\alpha} u_{\beta}$ is the projection tensor orthogonal to that vector, and $g_{\alpha \beta}$ is the Riemannian metric in the spacetime $\mathscr{M}$. As required by the homogeneity condition (3.137), the expression (3.140) gives a metric that depends on the direction, but not the magnitude, of the velocity. Eq. (3.140) may be compactly written as $\gamma_{\alpha \beta}=-p_{\alpha} p_{\beta}-h q_{\alpha \beta} p_{\gamma} u^{\gamma}$ where $p_{\alpha}=h u_{\alpha}+\eta_{\alpha}$. On shell, we have $u^{\alpha}=\dot{x}^{\alpha}$ and, by virtue of Eqs. (3.65) and (3.89), $\eta_{\alpha} u^{\alpha}=0$, i.e. the last term in Eq. (3.140) vanishes. The Finsler metric $\gamma_{\alpha \beta}$ plays the role of an effective metric felt by a magnetofluid element. Note that the 1-form $\eta_{\alpha}$, defined by Eq. (3.89) or (3.115), is consider independent of $\dot{x}^{\alpha}$. Therefore, the Lagrangian is linear in the velocity $\dot{x}^{\alpha}$ and the relevant Finsler space is of the Randers type [121, 122].

With the aid of Eqs. (3.135) and (3.140), the action functional (3.94) takes the form of the length (3.139). This functional is independent of $\tau$ and represents the arc length between events 1 and 2. That is, the motions of fluid elements in a barotropic, perfectly conducting flow are geodesics in a Finsler space with metric given by Eq. (3.140).

The geodesic equation is obtained by minimizing the functional (3.139) and using Eqs. (A7)- A10). This yields

$$
\frac{d^{2} x^{\lambda}}{d \tau^{2}}+\Gamma_{\mu \nu}^{\lambda} \frac{d x^{\mu}}{d \tau} \frac{d x^{\nu}}{d \tau}=0
$$

where

$$
\Gamma_{\mu \nu}^{\lambda}:=\frac{1}{2} \gamma^{\lambda \kappa}\left(\frac{\partial \gamma_{\kappa \mu}}{\partial x^{\nu}}+\frac{\partial \gamma_{\kappa \nu}}{\partial x^{\mu}}-\frac{\partial \gamma_{\mu \nu}}{\partial x^{\kappa}}\right)
$$

denote the Finslerian Christoffel symbols [119]. Although the above equations are identical to those of Riemannian geometry, the transformation law of the symbols $\Gamma_{\mu \nu}^{\lambda}$ is more complicated since it involves the Cartan torsion tensor:

$$
C_{\alpha \beta \gamma}:=\frac{1}{2} \frac{\partial \gamma_{\alpha \beta}}{\partial \dot{x}^{\gamma}}=\frac{3 h}{\left(-g_{\epsilon \zeta} \dot{x}^{\epsilon} \dot{x}^{\zeta}\right)^{1 / 2}} q_{(\alpha \beta} q_{\gamma) \delta} \eta^{\delta} .
$$

By extending the notion of a metric in $\mathcal{M}$ to allow for Finsler geometry, the problem of ideal MHD becomes one of pure geometry. We note that the geometry of the spacetime $\mathscr{M}$ remains Riemannian: no deviation from general relativity has been assumed. In the limit $\eta_{\alpha} \rightarrow$ 0 , the Cartan torsion tensor vanishes, the geometry of $\mathcal{M}$ also becomes Riemannian, and we recover the SyngeLichnerowicz result on barotropic fluids.

We note that a similar approach may be used for poorly conducting fluids, by replacing $\eta_{\alpha}$ with $e A_{\alpha}$ in the equations above, where $e$ is the net charge per fluid element [27]. Furthermore, with the replacements $h \rightarrow 1$, $\eta_{\alpha} \rightarrow e A_{\alpha}$, we recover the motion of a charged particle under the influence of an electromagnetic field in curved spacetime [121, 123 126]. We note, however, that for baroclinic fluids, the action is not parametrization invariant, and thus cannot be described within Riemann or Finsler geometry.

\section{DISCUSSION}

We have illustrated that barotropic flows and magnetoflows without viscosity, resistivity or other dissipation can be described via simple variational principles. These action principles can be written in terms of a Lagrangian density integrated over spacetime, as done traditionally for fluids, or in terms of a particle-like Lagrangian integrated over a proper-time or affine parameter. The latter approach paves the way for deriving simple Lagrangian and Hamiltonian descriptions of ideal $M H D$, in Newtonian and relativistic contexts. These descriptions are as valuable for fluids as they have been for classical mechanics and carry the same advantages over approaches focused on the equation-of-motion level.

For instance, certain conserved quantities - whose origin seems ad hoc when obtained by tedious algebraic manipulation of the equations of motion - emerge directly from the action in this geometric canonical approach. In particular, when the ideal MHD Lagrangians (3.71) and (3.120) admit continuous symmetries, Noether's theorem immediately yields the associated quantity conserved along streamlines [76]. As shown by Carter and Lichnerowicz, the relativistic hydrodynamics and magnetohydrodynamics are most naturally expressed in the language of differential forms. Cartan's identity can then be used to simplify calculations tremendously compared to the usual tensor or vector calculus, as demonstrated above. This approach to MHD is not yet very widely known, but this has been changing in recent years, and it is being used to obtain new results [8, 127 136]. For stationary and irrotational or corotating magnetoflows, Cartan's identity implies that these quantities, given by Eqs. (3.111) and (3.113), are constant throughout the fluid. These equations represent relativistic, magnetized generalizations of Bernoulli's principle and provide a way to construct equilibrium solutions via iterative methods 95, 137. Such results can be extended to the case of generalized Noether symmetries generated by Killing tensors (cf. 76] for details) and applied to the theory of black hole accretion rings [27, 47].

Several theoretical insights arise from this formulation. The symplectic geometry of phase space gives rise to various circulation theorems that stem from the PoincaréCartan integral invariant. The symplectic structure of the perfect MHD equations can be exploited in numerical simulations that use smoothed-particle hydrodynamic (SPH) methods [138]. For instance, symplectic or timesymetric methods can be used to conserve phase-space volume, circulation, and energy.

Geometric considerations have led to deeper understanding of magnetic phenomena in fluids in curved soacetime. Exploring the similarities of geodesic motion to hydrodynamic and magnetohydrodynamic motion, Lasota et al. [139] generalized the Penrose process 140] from point particles to fluid particles and jets. Moreover, the Finsler geometry described by the metric (3.140) allows one to represent ideal MHD flows as purely geodesic 
flows with no loss of generality. A notable feature of both pictures is that they are exact in time-dependent spacetimes, with gravitational and electromagnetic waves carrying energy and angular momentum away from the system. Although such geometrical insights have been sometimes used to construct first integrals for non-magnetized initial data [137], they have not so far been used for magnetized initial data or for evolving hydrodynamic and magnetohydrodynamic flows in numerical general relativity. The integrals (3.111), (3.113) and the evolution system 3.107 provide avenues for exploiting such geometric properties in the future.

\section{ACKNOWLEDGMENTS}

We thank Brandon Carter for pointing out the second line of Eq. (3.29) and a similar remark in the analogous equation for a fluid Killing tensor. We thank Theocharis Apostolatos, Jacob Bekenstein, Brandon Carter, Greg Comer, John Friedman, Roland Haas, David Hilditch, Darryl Holm, David Kaplan, Alan Kostelecky and Panagiotis Stavrinos for very fruitful discussions and comments. This work was supported by JSPS Grant-in-Aid for Scientific Research(C) 20540275, MEXT Grant-in-Aid for Scientific Research on Innovative Area 20105004, the Greek State Scholarships Foundation (IKY), NSF Grant PHY1001515, DFG grant SFB/Transregio 7 "Gravitational Wave Astronomy", STFC grant PP/E001025/1 and ANR grant 06-2-134423 Méthodes mathématiques pour la relativité générale. KU and EG acknowledge support from a JSPS Invitation Fellowship for Research in Japan (Short-term) and the invitation program of foreign researchers at the Paris observatory. CM and JPN thank the Paris Observatory for hospitality during the course of this work.

\section{Appendix A: Finsler geometry and Euler's theorem}

The homogeneity property 3.134 plays a fundamental role in Finsler geometry. This property gives rise to many important relations by means of Euler's homogeneous function theorem: Consider a function $Z(x, v)$ that is positively homogeneous of degree $r$ with respect to $v^{\alpha}$, that is,

$$
Z(x, \kappa v)=\kappa^{r} Z(x, v) \quad \forall \kappa>0 .
$$

Differentiating with respect to $\kappa$ and setting $\kappa=1$ yields

$$
v^{\alpha} \frac{\partial Z(x, v)}{\partial v^{\alpha}}=r Z(x, v) .
$$

This is the mathematical statement of Euler's theorem. Applying the above theorem to the case of the Lagrangian (3.134) yields

$$
\dot{x}^{\alpha} \frac{\partial L(x, \dot{x})}{\partial \dot{x}^{\alpha}}=L(x, \dot{x}) .
$$

Differentiating this expression with respect to $\dot{x}^{\alpha}$ yields

$$
\dot{x}^{\alpha} \frac{\partial^{2} L(x, \dot{x})}{\partial \dot{x}^{\alpha} \partial \dot{x}^{\beta}}=0 .
$$

Then, differentiating the relation

$$
\frac{1}{2} \frac{\partial L^{2}(x, \dot{x})}{\partial \dot{x}^{\alpha}}=L(x, \dot{x}) \frac{\partial L(x, \dot{x})}{\partial \dot{x}^{\alpha}}
$$

with respect to $\dot{x}^{\beta}$, contracting with $\dot{x}^{\alpha} \dot{x}^{\beta}$ and using Eqs. (A3) and (A4) yields

$$
L^{2}(x, \dot{x})=\underbrace{\frac{1}{2} \frac{\partial^{2} L^{2}(x, \dot{x})}{\partial \dot{x}^{\alpha} \partial \dot{x}^{\beta}}}_{-\gamma_{\alpha \beta}} \dot{x}^{\alpha} \dot{x}^{\beta} .
$$

Equations (A3) and (A6) reproduce (3.135). From Eqs. (3.134) and A6 we infer that the metric $\gamma_{\alpha \beta}(x, \dot{x})$ is homogeneous of degree zero in the velocity, Eq. (3.137). Then, applying Euler's theorem (A2) for $\gamma_{\alpha \beta}$ with $r=0$ yields

$$
\dot{x}^{\gamma} C_{\alpha \beta \gamma}=0
$$

where

$$
C_{\alpha \beta \gamma}:=\frac{1}{2} \frac{\partial \gamma_{\alpha \beta}}{\partial \dot{x}^{\gamma}}=\frac{1}{4} \frac{\partial^{3} L^{2}}{\partial \dot{x}^{\alpha} \partial \dot{x}^{\beta} \partial \dot{x}^{\gamma}}
$$

is the Cartan torsion tensor. The last equality, which follows from Eq. (3.136), implies that the above tensor is fully symmetric. From the above definition we infer that $C_{\alpha \beta \gamma}$ is homogeneous of degree $r=-1$ in the velocity. Then, Euler's theorem (A2) yields

$$
\dot{x}^{\delta} C_{\alpha \beta \gamma \delta}=-C_{\alpha \beta \gamma},
$$

where

$$
C_{\alpha \beta \gamma \delta}(x, \dot{x})=\frac{\partial C_{\alpha \beta \gamma}(x, \dot{x})}{\partial \dot{x}^{\delta}} .
$$

The geodesic equation in Finsler space can be obtained with the same variational methods as in a Riemann space, with additional use of Eqs. (A7)-A10. Finsler geometry reduces to Riemann geometry iff the Cartan torsion tensor and its derivatives vanish, whence the metric $\gamma_{\alpha \beta}$ is independent of velocity [119].

\section{Appendix B: Beckenstein-Oron current with one symmetry}

Assuming that the system obeys a Killing symmetry, i.e. that there exists a vector field $\overrightarrow{\boldsymbol{k}}$ such that

$$
\begin{aligned}
& £_{\overrightarrow{\boldsymbol{k}}} \boldsymbol{g}=0, \quad £_{\overrightarrow{\boldsymbol{k}}} \boldsymbol{u}=0, \quad £_{\overrightarrow{\boldsymbol{k}}} \boldsymbol{j}=0, \\
& £_{\overrightarrow{\boldsymbol{k}}} \boldsymbol{F}=0, \quad £_{\overrightarrow{\boldsymbol{k}}} h=0, \quad £_{\overrightarrow{\boldsymbol{k}}} \rho=0,
\end{aligned}
$$


a natural question is whether or not one can impose the same symmetry on the auxiliary quantities $\boldsymbol{a}$ and b. First, note that

$$
\begin{aligned}
£_{\overrightarrow{\boldsymbol{k}}} \boldsymbol{b} & =£_{\overrightarrow{\boldsymbol{k}}}(\overrightarrow{\boldsymbol{u}} \cdot \star \mathbf{d} \boldsymbol{a}) \\
& =\overrightarrow{\boldsymbol{u}} \cdot £_{\overrightarrow{\boldsymbol{k}}}(\star \mathbf{d} \boldsymbol{a}) \\
& =\overrightarrow{\boldsymbol{u}} \cdot \star £_{\overrightarrow{\boldsymbol{k}}}(\mathbf{d} \boldsymbol{a}) \text { (since } \overrightarrow{\boldsymbol{k}} \text { is Killing) } \\
& =\overrightarrow{\boldsymbol{u}} \cdot \star \mathbf{d}\left(£_{\overrightarrow{\boldsymbol{k}}} \boldsymbol{a}\right) \text { (since } \mathbf{d} \text { and } £_{\overrightarrow{\boldsymbol{k}}} \text { commute) }
\end{aligned}
$$

In addition, using equation (3.84), as well as the symmetries (B1), (B2),

$$
£_{\vec{k}} \mathbf{d} \boldsymbol{a}=£_{\overrightarrow{\boldsymbol{k}}}[\boldsymbol{u} \wedge \boldsymbol{B}+\star(\boldsymbol{u} \wedge \boldsymbol{b})]=\star\left(\boldsymbol{u} \wedge £_{\overrightarrow{\boldsymbol{k}}} \boldsymbol{b}\right) .
$$

We have therefore that

$$
£_{\overrightarrow{\boldsymbol{k}}} \boldsymbol{b}=0 \Leftrightarrow £_{\overrightarrow{\boldsymbol{k}}} \mathbf{d} \boldsymbol{a}=0 \Leftrightarrow £_{\overrightarrow{\boldsymbol{k}}} \boldsymbol{a} \text { closed }
$$

and of course $£_{\overrightarrow{\boldsymbol{k}}} \boldsymbol{a}=0$ implies $£_{\overrightarrow{\boldsymbol{k}}} \boldsymbol{b}=0$. So in effect, assuming that the auxiliary quantities $\boldsymbol{a}$ and $\boldsymbol{b}$ satisfy the same symmetry as the physical quantities is equivalent to assuming merely $£_{\overrightarrow{\boldsymbol{k}}} \boldsymbol{a}=0$. If on the other hand we are ready to sacrifice $£_{\overrightarrow{\boldsymbol{k}}} \boldsymbol{a}=0$ and to assume only that $£_{\overrightarrow{\boldsymbol{k}}} \boldsymbol{b}=0$, we must still impose that $£_{\overrightarrow{\boldsymbol{k}}} \boldsymbol{a}$ is closed.

We first notice that $£_{\vec{k}} \boldsymbol{a}=0$ is not systematically compatible with the gauge condition $\overrightarrow{\boldsymbol{u}} \cdot \boldsymbol{a}=0$. Indeed, let us consider the case where $\overrightarrow{\boldsymbol{u}}$ and $\overrightarrow{\boldsymbol{k}}$ are parallel, i.e.

$$
\overrightarrow{\boldsymbol{u}}=f \overrightarrow{\boldsymbol{k}}
$$

The question is whether we can impose consistently the three equations

$$
\begin{aligned}
\overrightarrow{\boldsymbol{u}} \cdot \boldsymbol{a} & =0, \\
£_{\overrightarrow{\boldsymbol{u}}} \boldsymbol{a} & =-\boldsymbol{B}, \\
£_{\overrightarrow{\boldsymbol{k}}} \boldsymbol{a} & =0 .
\end{aligned}
$$

Using the Cartan identity, we have

$$
-\boldsymbol{B}=£_{\overrightarrow{\boldsymbol{u}}} \boldsymbol{a}=f £_{\overrightarrow{\boldsymbol{k}}} \boldsymbol{a}+(\overrightarrow{\boldsymbol{k}} \cdot \boldsymbol{a}) \mathbf{d} f=(\overrightarrow{\boldsymbol{k}} \cdot \boldsymbol{a}) \mathbf{d} f=0
$$

since $\overrightarrow{\boldsymbol{u}} \cdot \boldsymbol{a}=0$ implies $\overrightarrow{\boldsymbol{k}} \cdot \boldsymbol{a}=0$. This is in general inconsistent.

Giving up the gauge condition $\overrightarrow{\boldsymbol{u}} \cdot \boldsymbol{a}=0$ does not improve things. Let us put $\phi=\overrightarrow{\boldsymbol{u}} \cdot \boldsymbol{a}$ and still assume that $\overrightarrow{\boldsymbol{u}}$ and $\overrightarrow{\boldsymbol{k}}$ are colinear. Now we have $£_{\overrightarrow{\boldsymbol{u}}} \boldsymbol{a}=\overrightarrow{\boldsymbol{u}} \cdot \mathbf{d} \boldsymbol{a}+\mathbf{d}(\overrightarrow{\boldsymbol{u}} \cdot \boldsymbol{a})$ and instead of (B10)-B12 we must consider

$$
\begin{aligned}
\overrightarrow{\boldsymbol{u}} \cdot \boldsymbol{a} & =\phi, \\
£_{\overrightarrow{\boldsymbol{u}}} \boldsymbol{a} & =-\boldsymbol{B}+\mathbf{d} \phi, \\
£_{\overrightarrow{\boldsymbol{k}}} \boldsymbol{a} & =0 .
\end{aligned}
$$

Then

$$
£_{\overrightarrow{\boldsymbol{u}}} \boldsymbol{a}=\phi \mathbf{d}(\log f)=-\boldsymbol{B}+\mathbf{d} \phi,
$$

i.e.

$$
\boldsymbol{B}=\phi \mathbf{d}\left(\log \left|\frac{\phi}{f}\right|\right)
$$

This forces the magnetic field $\boldsymbol{B}$ to be exact modulo multiplication by a scalar function, which is not a generic property. Indeed consider the 1 -form on $\mathbb{R}^{4}$

$$
\alpha=-y \mathbf{d} x+x \mathbf{d} y
$$

whose divergence vanishes. Can we find a globally defined smooth function $\psi$ such that $\psi \alpha$ be closed? This amounts to

$$
2 \psi+x \partial_{x} \psi+y \partial_{y} \psi=0
$$

which imposes that $\psi$ be homogeneous of degree -2 and contradicts the fact that $\psi$ be globally defined and smooth.

We conclude that we cannot in all generality assume that the auxiliary fields $\boldsymbol{a}$ and $\boldsymbol{b}$ satisfy the same symmetry as the physical quantities.

\section{Appendix C: Fluid super-Hamiltonians}

The canonical form of the Euler equation (3.13) involves only the thermodynamic variables $T, S, h$. We thus assert that the super-Hamiltonian for this equation has the general form

$$
H=H(h, S, T, N),
$$

where $N:=g^{\alpha \beta} p_{\alpha} p_{\beta}$ is the norm of the (generally nonnormalized) canonical momenta $p_{\alpha}$, whose nature is to be determined. Furthermore, we assume that the Hamiltonian generates a reparametrization with respect to the proper time of the fluid which we denote by a parameter $\mathrm{d} \lambda=\mathrm{d} \tau / \mathcal{A}$, where $\mathcal{A}$ is some function of the variables involved.

Computing Hamilton's equations and comparing them with the Euler equation we deduce that we are 'on-shell' only if $p_{\alpha}=h u_{\alpha}$ and thus $N=-h^{2}$. Additionally, the following equalities must be satisfied by the Hamiltonian on-shell in order to reproduce the Euler equation:

$$
\begin{aligned}
\frac{\partial H}{\partial T} & =0 \\
\frac{\partial H}{\partial h} & =2 h \frac{\partial H}{\partial N} \\
\frac{\partial H}{\partial S} & =-T \frac{\partial H}{\partial h}-2 T h \frac{\partial H}{\partial N} \\
\mathcal{A} & =2 h \frac{\partial H}{\partial N}
\end{aligned}
$$

One way to satisfy this set of constraints on the form of the Hamiltonian is via the expression

$$
H=\frac{\mathcal{C}^{\prime}(S)}{2 T h}\left(g^{\alpha \beta} p_{\alpha} p_{\beta}+h^{2}\right)-\mathcal{C}(S),
$$

where $\mathcal{C}(S)$ is an arbitrary function of the specific entropy with $\mathcal{C}^{\prime}(S) \neq 0$ for $S \geq 0$. The on-shell value of the conserved super-Hamiltonian is then $-\mathcal{C}(S)$ and 
the canonical time parameter $\lambda$ satisfies $\mathrm{d} \lambda=T \mathrm{~d} \tau / \mathcal{C}^{\prime}$. Carter's baroclinic Hamiltonian (3.125) is obtained simply by setting $\mathcal{C}(S)=S$.

For barotropic fluids one can use a similar approach to obtain a set of Hamiltonians of the form

$$
H=-\frac{\mathcal{D}(h)}{2 h}\left(g^{\alpha \beta} p_{\alpha} p_{\beta}+h^{2}\right)
$$

where $\mathcal{D}(h)$ is an arbitrary function of $h$, and the parametrization corresponding to this Hamiltonian is $\mathrm{d} \lambda=\mathrm{d} \tau / \mathcal{D}$. The transition between the Hamiltonians (C6) and (C7) for baroclinic and barotropic fluids depends on the form of temperature expressed as a function of entropy and enthalpy $T=T(h, S)$.

For baroclinic magnetofluids, we see from Eq. (3.93) that the streamlines of a perfectly conducting fluid behave as if under the influence of a vector potential $\boldsymbol{\eta}$. We thus assume that there is a canonical momentum $p_{\alpha}$ such that the Hamiltonian depends only on the normalization $N=g^{\alpha \beta}\left(p_{\alpha}-\kappa_{\alpha}\right)\left(p_{\beta}-\kappa_{\beta}\right)$ with $\kappa_{\alpha}$ some vector. In that case, we obtain the on-shell values $p_{\alpha}=h u_{\alpha}+\eta_{\alpha}, \kappa_{\alpha}=\eta_{\alpha} N=-h^{2}$ and the same set of constraints as in (C2)-C5. This means that one class of super-Hamiltonians which reproduce the ideal MHDEuler equation (3.93) is

$$
H=\frac{\mathcal{C}^{\prime}(S)}{2 T h}\left[g^{\alpha \beta}\left(p_{\alpha}-\eta_{\alpha}\right)\left(p_{\beta}-\eta_{\beta}\right)+h^{2}\right]-\mathcal{C}(S),
$$

where the on-shell value of the super-Hamiltonian is again $-\mathcal{C}(S)$ and the canonical time parameter $\lambda$ satisfies $\mathrm{d} \lambda=T \mathrm{~d} \tau / \mathcal{C}^{\prime}$.

[1] M. Mościbrodzka, C. F. Gammie, J. C. Dolence, H. Shiokawa, and P. K. Leung, Astrophys. J. 706, 497 (2009).

[2] Z. Meliani, C. Sauty, K. Tsinganos, E. Trussoni, and V. Cayatte, Astron. Astrophys. 521, A67 (2010)

[3] S. S. Komissarov, in Mem SAIt, Vol. 82 (2011) pp. 95-103.

[4] V. Beskin, MHD Flows in Compact Astrophysical Objects: Accretion, Winds and Jets (Springer, 2009).

[5] H. Sotani, A. Colaiuda, and K. D. Kokkotas, Mon. Not. R. Astron. Soc. 385, 2161 (2008).

[6] H. Sotani, K. D. Kokkotas, and N. Stergioulas, Mon. Not. R. Astron. Soc. Lett. 385, L5 (2008)

[7] B. Zink, P. D. Lasky, and K. D. Kokkotas, Phys. Rev. D 85, 024030 (2012)

[8] M. Freytsis and S. E. Gralla, J. Cosmol. Astropart. Phys. 2016, 042 (2016).

[9] A. L. Watts, N. Andersson, D. Chakrabarty, M. Feroci, K. Hebeler, G. Israel, F. K. Lamb, M. C. Miller, S. Morsink, F. Özel, A. Patruno, J. Poutanen, D. Psaltis, A. Schwenk, A. W. Steiner, L. Stella, L. Tolos, and M. van der Klis, Rev. Mod. Phys. 88, 021001 (2016).

[10] A. Lichnerowicz, Relativistic hydrodynamics and magnetohydrodynamics (W. A. Benjamin, New York, NY, 1967).

[11] J. A. Font, Living Rev. Relativ. 11 (2008).

[12] L. Antón, J. Miralles, J. Martí, J. Ibanez, M. Aloy, and P. Mimica, Astrophys. J. Suppl. 188, 1 (2010).

[13] K. Glampedakis, N. Andersson, and S. K. Lander, Mon. Not. R. Astron. Soc. 420, 1263 (2012)

[14] N. Andersson, I. Hawke, K. Dionysopoulou, and G. L. Comer, (2016) arXiv:1610.00448.

[15] N. Andersson, K. Dionysopoulou, I. Hawke, and G. L. Comer, (2016) arXiv:1610.00449.

[16] A. Reisenegger, Astron. Astrophys. 499, 557 (2009).

[17] J. L. Synge, London Math 43 (2002).

[18] A. Lichnerowicz, Ann. Sci. École Norm. Sup 58, 285 (1941)

[19] V. Arnold, Ann. l'institut Fourier 16, 319 (1966).

[20] D. D. Holm, J. E. Marsden, T. Ratiu, and A. Weinstein, Phys. Rep. 123, 1 (1985).

[21] D. D. Holm, Phys. D Nonlinear Phenom. 17, 1 (1985)

[22] D. D. Holm, Phys. D Nonlinear Phenom. 25, 261 (1987)

[23] D. D. Holm, J. E. Marsden, and T. S. Ratiu, Adv. Math. (N. Y). 137, 1 (1998).

[24] D. D. Holm, Geometric Mechanics (Part I: Dynamics and Symmetry), 2nd ed. (Imperial College Press, Oxford, 2011).

[25] D. D. Holm, Geometric Mechanics (Part II: Rotating, Translating and Rolling ) 2nd ed. (Imperial College Press, Oxford, 2011).

[26] T. Tao, Compactness and contradiction (AMS, 2013) pp. 205-206.

[27] B. Carter, in Act. Galact. Nucl., edited by C. Hazard and S. Mitton (Cambridge University Press, Cambridge, 1979) p. 273.

[28] C. Markakis and L. Barack, , 9 (2014), arXiv:1406.4865.

[29] J. D. Bekenstein and A. Oron, Phys. Rev. E 62, 5594 (2000)

[30] J. Bekenstein and A. Oron, Found. Phys. 31, 895 (2001).

[31] J. Bekenstein and G. Betschart, Phys. Rev. D 74, 083009 (2006).

[32] J. D. Bekenstein, Astrophys. J. 319, 207 (1987).

[33] C. Markakis, J. S. Read, M. Shibata, K. Uryu, J. D. E. Creighton, J. L. Friedman, and B. D. Lackey, in J. Phys. Conf. Ser. Vol. 189, edited by N. Stergioulas (IOP Science, Philadelphia, 2009) p. 012024.

[34] J. S. Read, C. Markakis, M. Shibata, K. Uryu, J. D. E. Creighton, and J. L. Friedman, Phys. Rev. D 79, 12 (2009).

[35] C. Markakis, J. S. Read, M. Shibata, K. Uryu, J. D. E. Creighton, and J. L. Friedman, in 12th Marcel Grossman Meet. edited by T. Damour, R. Jantzen, and R. Ruffini (World Scientific, Singapore, 2011) pp. 5-7. 
[36] W. E. East, F. Pretorius, and B. C. Stephens, Phys. Rev. D 85, 124010 (2012).

[37] B. D. Farris, R. Gold, V. Paschalidis, Z. B. Etienne, and S. L. Shapiro, Phys. Rev. Lett. 109, 221102 (2012)

[38] B. Giacomazzo, L. Rezzolla, and L. Baiotti, Phys. Rev. D 83, 044014 (2011).

[39] J. S. Read, L. Baiotti, J. D. E. Creighton, J. L. Friedman, B. Giacomazzo, K. Kyutoku, C. Markakis, L. Rezzolla, M. Shibata, and K. Taniguchi, Phys. Rev. D 88, 044042 (2013).

[40] P. Mosta, B. C. Mundim, J. A. Faber, R. Haas, S. C. Noble, T. Bode, F. Löffler, C. D. Ott, C. Reisswig, and E. Schnetter, Class. Quantum Gravity 31, 015005 (2014).

[41] K. Dionysopoulou, D. Alic, and L. Rezzolla, Phys. Rev. D 92, 084064 (2015).

[42] T. Kawamura, B. Giacomazzo, W. Kastaun, R. Ciolfi, A. Endrizzi, L. Baiotti, and R. Perna, Phys. Rev. D 94, $064012(2016)$

[43] I. Contopoulos, J. Plasma Phys. 82, 1 (2016)

[44] A. Nathanail, A. Strantzalis, and I. Contopoulos, Mon. Not. R. Astron. Soc. 455, 4479 (2016)

[45] O. Zanotti, L. Rezzolla, L. Del Zanna, and C. Palenzuela, Astron. Astrophys. 523, A8 (2010)

[46] O. Korobkin, E. Abdikamalov, N. Stergioulas, E. Schnetter, B. Zink, S. Rosswog, and C. D. Ott, Mon. Not. R. Astron. Soc. 431, 349 (2013)

[47] I. Contopoulos, A. Nathanail, and M. Katsanikas, Astrophys. J. 805, 105 (2015).

[48] A. P. Kouretsis and C. G. Tsagas, Phys. Rev. D 82, 124053 (2010)

[49] A. P. Kouretsis and C. G. Tsagas, Phys. Rev. D 88, 044006 (2013).

[50] J. D. Barrow and C. G. Tsagas, Mon. Not. R. Astron. Soc. 414, 512 (2011)

[51] C. G. Tsagas, Phys. Rev. D 84, 043524 (2011).

[52] F. Dosopoulou, F. Del Sordo, C. G. Tsagas, and A. Brandenburg, Phys. Rev. D 85, 063514 (2012)

[53] J. D. Barrow, C. G. Tsagas, and K. Yamamoto, Phys. Rev. D 86, 023533 (2012)

[54] J. D. Barrow, C. G. Tsagas, and K. Yamamoto, Phys. Rev. D 86, 107302 (2012)

[55] F. Dosopoulou and C. G. Tsagas, Phys. Rev. D 89, 103519 (2014)

[56] U.-L. Pen and N. Turok, Phys. Rev. Lett. 117, 131301 (2016)

[57] E. Gourgoulhon, C. Markakis, K. Uryu, and Y. Eriguchi, Phys. Rev. D 83, 104007 (2011).

[58] E. Gourgoulhon, in EAS Publ. Ser., Vol. 21 (M. Rieutord and B. Dubrulle, 2006) pp. 43-79.

[59] E. Gourgoulhon, Special Relativity in General Frames: From Particles to Astrophysics (Springer, Paris, 2013).

[60] J. L. Friedman and N. Stergioulas, Rotating Relativistic Stars (Cambridge University Press, New York, NY, 2013).

[61] R. Abraham and J. E. Marsden, Foundations of mechanics (AMS Chelsea Pub., 2008) p. 826.

[62] H. Poincaré, Acta Math. 13, 1 (1890).

[63] H. Poincaré, in Gauthier-Villars, Vol. 3 (1899) p. Chapt. 26.

[64] É. Cartan, Leçons sur les invariants intégraux (Herman, Paris, 1922).

[65] D. Boccaletti and G. Pucacco, Theory of Orbits: Volume 1 (Springer, 2003).

[66] C. M. Markakis, "Hamiltonian Hydrodynamics and Irrotational Binary Inspiral," (2014), arXiv:1410.7777.

[67] H. Ertel and C.-G. Rossby, Geofis. Pura e Appl. 14, 189 (1949).

[68] A. F. Cheviakov and M. Oberlack, J. Fluid Mech. 760, 368 (2014).

[69] H. Alfvén, Nature 150, 405 (1942)

[70] L. Woltjer, Proc. NUI. Acad. Sci. 44, 833 (1958).

[71] H. K. Moffatt, J. Fluid Mech. 35, 117 (1969).

[72] B. Carter and I. Khalatnikov, Ann. Phys. (N. Y). 219, 243 (1992)

[73] P. J. Ioannou and T. A. Apostolatos, Elements of Theoretical Mechanics (in Greek), 1st ed. (Leader Books, Athens, 2004).

[74] A. H. Taub, Ration. Mech. Anal 3 (1959).

[75] D. Christodoulou, Bull. Am. Math. Soc. 44, 581 (2007).

[76] C. Markakis, Rotating and binary neutron stars with magnetic fields (PhD Dissertation, University of WisconsinMilwaukee, 2011).

[77] A.-L. Cauchy, Théorie de la propagation des ondes à la surface d'un fluide pesant d'une profondeur indéfinie, tome i ed. (1815) pp. 5-318.

[78] U. Frisch and B. Villone, Eur. Phys. J. H 39, 325 (2014).

[79] W. Thomson, Trans. Roy. Soc. Edinburgh. 25, 217 (1869).

[80] A. Lichnerowicz, Théories Relativistes de la Gravitation et de L'Electromagnetisme (Masson Cie, Paris, 1955).

[81] R. Prix, Phys. Rev. D 69, 043001 (2004).

[82] R. Prix, Phys. Rev. D 71, 083006 (2005)

[83] C. W. Misner, K. S. Thorne, and . J. A. Wheeler, Gravitation (Freeman, New York, 1973).

[84] R. M. Wald, General Relativity (University of Chicago Press, Chicago, 1984).

[85] C. Markakis, Mon. Not. R. Astron. Soc. 441, 2974 (2014).

[86] X. Huang, C. Markakis, N. Sugiyama, and K. Uryu, Phys. Rev. D 78, 124023 (2008)

[87] S. Bonazzola, E. Gourgoulhon, and J.-A. Marck, Phys. Rev. D 56, 7740 (1997)

[88] S. A. Teukolsky, Astrophys. J. 504, 442 (1998).

[89] P. Marronetti, G. J. Mathews, and J. R. Wilson, Phys. Rev. D 60, 4 (1999).

[90] E. Gourgoulhon, arXiv:9804054 [gr-qc]

[91] M. Shibata, Phys. Rev. D 58, 024012 (1998)

[92] S. Bonazzola, E. Gourgoulhon, and J.-A. Marck, Phys. Rev. Lett. 82, 892 (1999). 
[93] K. Uryu and Y. Eriguchi, Phys. Rev. D 61, 124023 (2000)

[94] K. Taniguchi and M. Shibata, Astrophys. J. Suppl. Ser. 188, 187 (2010)

[95] R. H. Price, C. Markakis, and J. L. Friedman, J. Math. Phys. 50, 16 (2009).

[96] P. Marronetti and S. L. Shapiro, Phys. Rev. D 68, 104024 (2003)

[97] W. Tichy, Phys. Rev. D 84, 024041 (2011)

[98] W. Tichy, Phys. Rev. D 86, 064024 (2012)

[99] W. Tichy, Reports Prog. Phys. 80 (2016).

[100] N. Moldenhauer, C. M. Markakis, N. K. Johnson-McDaniel, W. Tichy, and B. Brügmann, Phys. Rev. D 90, 084043 (2014),

[101] T. Dietrich, N. Moldenhauer, N. K. Johnson-McDaniel, S. Bernuzzi, C. M. Markakis, B. Brügmann, and W. Tichy, Phys. Rev. D 92, 124007 (2015).

[102] T. Padmanabhan, Gravitation: Foundations and Frontiers (Cambridge University Press, 2010) p. 728.

[103] L. D. Landau, The classical theory of fields, 4th ed. (Pergamon Press, 1975).

[104] J. L. Friedman, Commun. Math. Phys. 62, 247 (1978).

[105] J. Katz, Proc. R. Soc. London A Math. Phys. Eng. Sci. 391 (1984).

[106] B. Carter, "Covariant theory of conductivity in ideal fluid or solid media," in Relativ. Fluid Dyn., edited by A. M. Anile and Y. Choquet-Bruhat (Springer, Berlin, Heidelberg, 1989) pp. 1-64.

[107] Z. B. Etienne, Y. T. Liu, and S. L. Shapiro, Phys. Rev. D 82, 084031 (2010).

[108] Z. B. Etienne, V. Paschalidis, Y. T. Liu, and S. L. Shapiro, Phys. Rev. D 85, 024013 (2012).

[109] C. F. Gammie, J. C. McKinney, and G. Tóth, Astrophys. J. 589, 444 (2003).

[110] J. Frauendiener, Class. Quantum Gravity 20, L193 (2003)

[111] R. A. Walton, Houst. J. Math. 31, 145 (2005).

[112] T. A. Oliynyk, E. L. C, and D. W.-M. Choquet-Bruhat Y, Class. Quantum Gravity 29, 155013 (2012).

[113] T. A. Oliynyk, Bull. des Sci. Mathématiques 141, 105 (2017), arXiv:1501.00045.

[114] K. Uryu, E. Gourgoulhon, and C. Markakis, Phys. Rev. D 82, 104054 (2010).

[115] C. Markakis, K. Uryu, and E. Gourgoulhon, in J. Phys. Conf. Ser. 283, edited by L. Perivolaropoulos (IOP Science, Philadelphia, 2011) p. 012021.

[116] K. Uryu, E. Gourgoulhon, C. M. Markakis, K. Fujisawa, A. Tsokaros, and Y. Eriguchi, Phys. Rev. D 90, 101501 (2014)

[117] B. Haskell, L. Samuelsson, K. Glampedakis, and N. Andersson, Not. R 385 (2008).

[118] S.-S. Chern, Not. Am. Math. Soc. 43, 959 (1996).

[119] G. S. Asanov, Finsler Geometry, Relativity and Gauge Theories (Springer, 1985).

[120] D. Lovelock and H. Rund, Tensors, Differential Forms, and Variational Principles (Dover Publications, 1989).

[121] G. Randers, Phys. Rev. 59, 195 (1941)

[122] S. Basilakos, A. P. Kouretsis, E. N. Saridakis, and P. C. Stavrinos, Phys. Rev. D 88, 123510 (2013)

[123] G. S. Asanov, Math. Phys 11, 221 (1977).

[124] G. Gibbons and C. Warnick, Contemp. Phys. 52, 197 (2011)

[125] G. Gibbons, C. Herdeiro, C. Warnick, and M. Werner, Phys. Rev. D 79, 37 (2009).

[126] G. W. Gibbons and C. M. Warnick, Ann. Phys. (N. Y). 325, 909 (2010).

[127] T. D. Brennan, S. E. Gralla, and T. Jacobson, Class. Quantum Gravity 30, 195012 (2013)

[128] S. E. Gralla and T. Jacobson, Mon. Not. R. Astron. Soc. 445, 2500 (2014)

[129] T. D. Brennan and S. E. Gralla, Phys. Rev. D 89, 103013 (2014).

[130] S. E. Gralla and T. Jacobson, Phys. Rev. D 92, 043002 (2015).

[131] S. E. Gralla, A. Lupsasca, and M. J. Rodriguez, Phys. Rev. D 92, 044053 (2015)

[132] S. E. Gralla and P. Zimmerman, Phys. Rev. D 93, 123016 (2016).

[133] S. E. Gralla, A. Lupsasca, and M. J. Rodriguez, Phys. Rev. D 93, 044038 (2016)

[134] S. E. Gralla, A. Lupsasca, and A. Strominger, Phys. Rev. D 93, 104041 (2016)

[135] S. E. Gralla, A. Lupsasca, and A. Philippov, (2016), arXiv:1604.04625

[136] G. Compère, S. E. Gralla, and A. Lupsasca, (2016), arXiv:1606.06727.

[137] E. Gourgoulhon, (2010), arXiv:1003.5015.

[138] S. Rosswog, Living Rev. Comput. Astrophys. 1, 1 (2015)

[139] J.-P. Lasota, E. Gourgoulhon, M. Abramowicz, A. Tchekhovskoy, and R. Narayan, Phys. Rev. D 89, 024041 (2014).

[140] R. Penrose and R. M. Floyd, Nat. Phys. Sci. 229, 177 (1971) 